\title{
Hybrid Global Matrix/Local Interaction Simulation Approach for Wave Propagation in Composites
}

\author{
Matthew B. Obenchain,,$*$ Kalyan S. Nadella,,$*$ and Carlos E. S. Cesnik \\ University of Michigan, Ann Arbor, Michigan 48109 \\ DOI: $\underline{10.2514 / 1 . J 053101}$
}

\begin{abstract}
This paper presents a hybrid approach to model guided wave propagation in composite laminates. The global matrix approach is used to determine the displacement field surrounding a piezoelectric actuator. The displacement field is then enforced in a specified region of a numerical model that employs the local interaction simulation approach. This hybrid approach improves upon previous local interaction simulation approach methods that prescribe in-plane displacements within the actuator profile to model actuation. Additionally, it circumvents the problem of modeling nonrectangular actuators in the Cartesian discretization in the local interaction simulation approach. Results show that the hybrid model outperforms previous local interaction simulation approach models when considering actuation from circular, square, and composite long-range variable-direction emitting radar actuators. The hybrid model produces wave propagation time histories that closely match the baseline global matrix method and successfully capture directional effects resulting from the anisotropic nature of composite plates. The dependence of the results on in-plane discretization size is examined for aluminum, cross-ply, unidirectional, and quasi-isotropic plates. The results for the aluminum and unidirectional plates show considerable dependence on the in-plane cell dimensions, but that dependence is less pronounced for the cross-ply and quasi-isotropic cases.
\end{abstract}

\begin{tabular}{|c|c|c|}
\hline$A_{0}$ & $=$ & fundamental antisymmetric Lamb wave mode \\
\hline$A_{1}, A_{2}$ & $=$ & in-plane dimensions of rectangular actuator, $\mathrm{m}$ \\
\hline & $=$ & vector of displacement amplitude constants \\
\hline$C$ & $=$ & displacement amplitude constant \\
\hline$c$ & $=$ & phase velocity, $\mathrm{m} / \mathrm{s}$ \\
\hline$c_{M}$ & $=$ & Fourier series coefficient \\
\hline$E, E_{1}, E_{2}$ & $=$ & moduli of elasticity, $\mathrm{Pa}$ \\
\hline $\boldsymbol{E}_{u}, \boldsymbol{E}_{d}$ & $=$ & global matrix exponential matrices \\
\hline $\boldsymbol{e}$ & $=$ & displacement eigenvector \\
\hline $\boldsymbol{F}$ & $=$ & vector of traction components from actuator \\
\hline$F_{1}, F_{2}$ & $=$ & traction components from actuator \\
\hline$G_{12}, G_{23}$ & $=$ & shear moduli, $\mathrm{Pa}$ \\
\hline$H$ & $=$ & Hankel function \\
\hline$i$ & $=$ & imaginary number \\
\hline$J$ & $=$ & Bessel function \\
\hline$K$ & $=$ & global radial wavenumber, $1 / \mathrm{m}$ \\
\hline$K_{1}, K_{2}$ & $=$ & $\begin{array}{l}\text { in-plane components of global radial wave- } \\
\text { number, } 1 / \mathrm{m}\end{array}$ \\
\hline $\boldsymbol{L}^{m}$ & $=$ & coordinate transformation matrix for $m$ th lamina \\
\hline$M$ & $=$ & Fourier series index \\
\hline $\boldsymbol{Q}_{i j}^{m}$ & $=$ & $\begin{array}{l}\text { matrix of stress and displacement eigenvectors for } \\
m \text { th lamina }\end{array}$ \\
\hline$R$ & $=$ & radius of circular actuator, $\mathrm{m}$ \\
\hline$r$ & $=$ & radial position, $\mathrm{m}$ \\
\hline$S$ & $=$ & stiffness matrix \\
\hline$S_{i j}$ & $=$ & stiffness matrix component \\
\hline$S_{0}$ & $=$ & fundamental symmetric Lamb wave mode \\
\hline
\end{tabular}

Presented as Paper 2013-1658 at the 54th AIAA/ASME/ASCE/AHS/ASC Structures, Structural Dynamics, and Materials Conference, Boston, MA, 811 April 2013; received 15 October 2013; revision received 17 January 2014; accepted for publication 20 January 2014; published online 9 May 2014. Copyright $\odot 2014$ by Matthew B. Obenchain, Kalyan S. Nadella, and Carlos E. S. Cesnik. Published by the American Institute of Aeronautics and Astronautics, Inc., with permission. Copies of this paper may be made for personal or internal use, on condition that the copier pay the $\$ 10.00$ per-copy fee to the Copyright Clearance Center, Inc., 222 Rosewood Drive, Danvers, MA 01923; include the code 1533-385X/14 and $\$ 10.00$ in correspondence with the CCC.

*Ph.D. Candidate, Department of Aerospace Engineering, 1320 Beal Avenue. Member AIAA.

${ }^{\dagger}$ Professor, Department of Aerospace Engineering, 1320 Beal Avenue; cesnik@umich.edu. Fellow AIAA (Corresponding Author).

\begin{tabular}{|c|c|c|}
\hline$t$ & $=$ & time or thickness, $\mathrm{s}, \mathrm{m}$ \\
\hline $\boldsymbol{U}$ & $=$ & displacement vector in wavenumber domain \\
\hline$U_{i}$ & $=$ & $\begin{array}{l}\text { displacement component in wavenumber domain } \\
\text { in } i \text { th direction }\end{array}$ \\
\hline$u$ & $=$ & displacement vector in spatial domain \\
\hline$u$ & $=$ & $\begin{array}{l}\text { displacement component in spatial domain in } 1 \text { - } \\
\text { direction, } m\end{array}$ \\
\hline$u_{i}$ & $=$ & $\begin{array}{l}\text { displacement component in spatial domain in } i \\
\text { direction, } \mathrm{m}\end{array}$ \\
\hline$v$ & $=$ & $\begin{array}{l}\text { displacement component in spatial domain in 2- } \\
\text { direction, } m\end{array}$ \\
\hline$w$ & $=$ & $\begin{array}{l}\text { displacement component in spatial domain in 3- } \\
\text { direction (out-of-plane), } \mathrm{m}\end{array}$ \\
\hline$X_{i}$ & $=$ & $\begin{array}{l}\text { position component in global coordinate system, } \\
\mathrm{m}\end{array}$ \\
\hline$x_{i}$ & $=$ & position component in local coordinate system, $\mathrm{m}$ \\
\hline$\alpha, \beta, \gamma$ & $=$ & $\begin{array}{l}\text { local interaction simulation approach position } \\
\text { indices }(1,-1)\end{array}$ \\
\hline$\Gamma$ & $=$ & global azimuthal wavenumber, $1 / \mathrm{m}$ \\
\hline $\begin{array}{l}\Delta x_{1} \\
\Delta x_{2}, \Delta x_{3}\end{array}$ & $=$ & $\begin{array}{l}\text { spatial discretizations in local interaction } \\
\text { simulation approach, } m\end{array}$ \\
\hline$\Delta t$ & $=$ & $\begin{array}{l}\text { time step in local interaction simulation approach, } \\
\mathrm{s}\end{array}$ \\
\hline$\Delta$ & $=$ & determinant of global matrix \\
\hline$\zeta$ & $=$ & local through-thickness wavenumber, $1 / \mathrm{m}$ \\
\hline$\eta_{x}, \eta_{y}, \eta_{z}$ & $=$ & $\begin{array}{l}\text { reciprocals of local interaction simulation } \\
\text { approach spatial discretizations, } 1 / \mathrm{m}\end{array}$ \\
\hline$\theta$ & $=$ & azimuthal position \\
\hline$\nu$ & $=$ & Poisson's ratio \\
\hline$\xi$ & $=$ & local in-plane wavenumber, $1 / \mathrm{m}$ \\
\hline$\rho$ & $=$ & density, $\mathrm{kg} / \mathrm{m}^{3}$ \\
\hline$\tau_{0}$ & $=$ & stress amplitude constant \\
\hline$\chi$ & $=$ & $\begin{array}{l}\text { local interaction simulation approach parameter } \\
\text { comparing time step and material density, } \mathrm{s}^{2} \text {. } \\
\mathrm{m}^{3} / \mathrm{kg}\end{array}$ \\
\hline$\Psi$ & $=$ & global matrix displacement function \\
\hline & $=$ & requency, $1 / \mathrm{s}$ \\
\hline
\end{tabular}

\section{Superscripts}

$\begin{array}{lll}d & = & \text { downward } \\ i, j, k & = & \text { direction indices }(1,2,3) \\ m & = & \text { lamina number } \\ N & = & \text { number of layers }\end{array}$




$\begin{array}{lll}T & =\text { matrix transpose } \\ t & =\text { time, } \mathrm{s} \\ u & =\text { upward } \\ \alpha, \beta, \gamma & =\quad \begin{array}{l}\text { local interaction simulation approach position } \\ \end{array} & \text { indices }(1,-1)\end{array}$

\section{Subscripts}

$\begin{array}{lll}d & = & \text { downward } \\ S & = & \text { symmetric lay-up } \\ u & & =\end{array}$

\section{Introduction}

$\mathbf{U}$ SE of composite materials has proliferated in the aerospace community in recent years because of their significant advantages over conventional metals such as high specific strength, high specific stiffness, and long fatigue life. Appropriate structural health monitoring (SHM) strategies are required to ensure the functionality and reliability of composite structures. In the past, numerous studies [1] have shown the use of guided waves $(\mathrm{GWs})$ to be an efficient method for damage detection in metallic structures, which motivates similar research in the field of composite materials. Because of the anisotropic nature of composite materials, the development of SHM methods is significantly more complicated and challenging than in the case of isotropic materials. A comprehensive understanding of the GW propagation behavior is required to develop reliable structural health monitoring systems.

Previous researchers have modeled GW propagation in composites using a wide range of analytical, semi-analytical, and numerical approaches. Purely analytical models for wave propagation in composites have been limited. For example, Ditri and Rose [2] used a normal mode-expansion technique to model excitation of anisotropic layers, but it was based on a two-dimensional (2-D) elasticity model. Datta et al. [3] highlighted the use of approximate plate theories due to the complexity of the equations obtained when a full threedimensional (3-D) theory of elasticity is used with layered structures. The most successful theoretical strategies for modeling wave propagation in multilayered plates have been the matrix approaches: the transfer matrix method and the global matrix method [4].

Researchers have used the global matrix method as the basis for a semi-analytical formulation to study the GW excitation and propagation produced by finite-dimensional piezoelectric-based transducers [5-7]. In the majority of these studies, the goal has been to use a theoretical approach to determine dispersion behavior or out-ofplane displacement values for comparison with experiments using laser vibrometry or other measurement techniques.

Although theoretical models are capable of characterizing the GW propagation in an infinite medium, they often become intractable when modeling complex composite structures because of the interface conditions and the need to account for the shape and reflections in the formulation. Researchers have turned to numerical simulations to facilitate the modeling of waves, which can better deal with complex structural systems, interfaces, and damage [8]. Lee and Staszewski [9] provided a good review of the many different numerical techniques used to model $\mathrm{GW}$, including the finite difference (FD) approach, the finite element method, the boundary element method, and spectral element methods. Although numerical tech- niques for modeling wave propagation are varied and have been successful, many have proved to be high in computational cost [1].

The local interaction simulation approach (LISA), originally developed by Delsanto et al. [10-12], has emerged as an efficient numerical option for modeling $\overline{\mathrm{GW}}$. This method is based on a set of iterative equations for "unit cells" that discretize the structure, and it uses a sharp interface model to handle material discontinuities and layer interfaces. Recent implementations $[13,14]$ of this method have shown good results, but two main difficulties have surfaced. First, current LISA implementations have been limited to rectangular or cubic Cartesian grids, which create a challenge when modeling circular actuators. It would be advantageous to have a method that incorporates the behavior of the actuator without relying on the geometry of the grid in LISA. Second, most of the current implementations model the actuators by specifying in-plane displacements on the surface of the plate within the profile of the actuator. As this paper will demonstrate, this approach leads to inaccuracies in the displacement time histories even at points away from the actuator. An alternative method to capture the behavior of the actuator is desirable.

The aim of this paper is to implement a hybrid approach to modeling wave propagation in composite laminates. The global matrixbased semi-analytical method is used to determine the complete displacement time history for a set of points located near a piezoelectric actuator both at the surface and through the thickness dimension of the composite plate. Subsequently, these known displacements are specified in LISA to represent the actuation pulse, and the remainder of the wave propagation model is obtained numerically. This provides a seamless way to integrate nonrectangular actuators into the Cartesian grid of the LISA simulation, allowing for an accurate representation of the wave field and avoiding errors associated with the actuator discretization. Additionally, it correctly represents the tractions applied by any actuator geometry (including rectangular) and avoids the need to assume a pattern for prescribed displacements in the actuator region.

\section{Local Interaction Simulation Approach Hybrid Model}

This section describes the overarching structure of the LISA hybrid model. As mentioned previously, the global matrix method is well suited to capture the effects of finite-dimensional actuators, but it cannot capture damage or lateral plate boundaries. On the other hand, LISA can successfully model a finite-dimensional plate, but representing the effects of surface-mounted actuators is challenging. As an alternative, the LISA hybrid model is introduced that leverages the strengths of both methods. It uses the global matrix approach to represent the effects of the actuator while using LISA for the majority of the plate structure.

First, the overall LISA model is defined according to the structure of the plate, including any material differences between layers for a laminate. As in previous LISA models, air cells are included at the plate boundaries to allow for traction-free boundary conditions. Next, a cutout region around the actuator is defined as in Fig. 1, and the nodes within the LISA model located on the boundary of this region are identified and tabulated.

Third, the time histories of the three displacement components resulting from the piezoelectric actuation are calculated at each of the cutout boundary points using the global matrix method. Because the entire time history of the displacements is calculated and stored, this

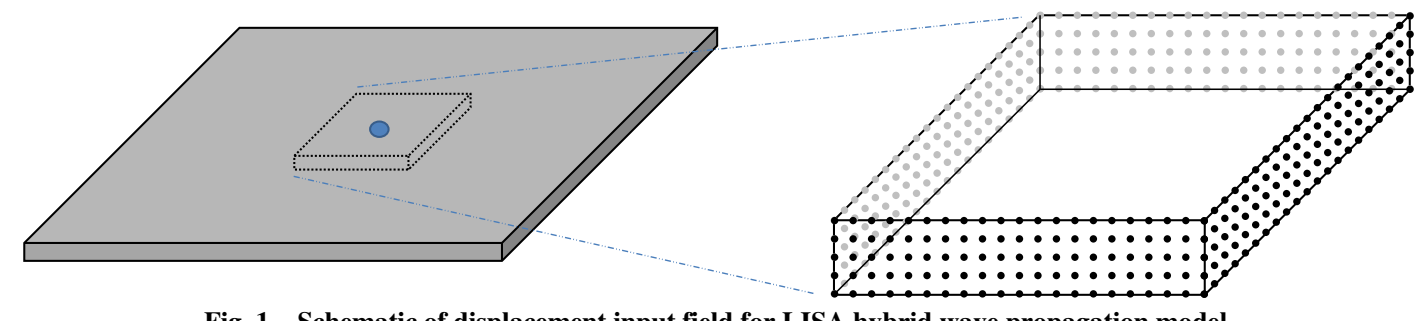

Fig. 1 Schematic of displacement input field for LISA hybrid wave propagation model. 
step only needs to be run once for the plate configuration used in the simulation. Finally, the square cutout region surrounding the actuator is segregated from the remainder of the model, and the LISA simulation is started. At each time step in the LISA simulation, the displacement values for the boundary nodes of this region are specified according to the global matrix time history. The LISA model is run as usual to completely capture the wave propagation behavior for the remainder of the plate.

Although the LISA hybrid model still specifies displacements, the actuation modeling method is distinct from previous LISA implementations. Because the LISA hybrid model uses the global matrix method to capture the behavior of the actuator, it inherently assumes the actuator imposes tractions, not displacements, on the plate surface. These tractions are included in the solution process used to determine the displacement time histories at all points along cutout boundaries.

The next two sections describe the two major components of the hybrid model in detail. First, the global matrix method is explained, including the steps needed to represent the laminated structure of a composite plate and the approach used to model the actuator. Second, the fundamentals of LISA and previous actuator modeling strategies are presented as background.

\section{Global Matrix Method}

\section{A. Theoretical Development}

Raghavan and Cesnik [6] outlined a suitable framework to determine the displacements resulting from a piezoelectric actuator attached to the surface of a composite laminate. A block diagram of this process is given as Fig. 2. The starting point for the analysis is the displacement form of the equilibrium equation for a transversely isotropic bulk medium:

$$
\nabla \boldsymbol{S} \nabla^{T} \boldsymbol{u}=\rho \ddot{\boldsymbol{u}}
$$

where $\boldsymbol{S}$ is the stiffness matrix, $\boldsymbol{u}$ is the displacement vector, and $\rho$ is the media density.

Assumption of a plane wave solution for Eq. (1) results in an eigenvalue problem that can be solved to yield a displacement field of the form

$$
\begin{aligned}
\boldsymbol{u}= & \left(C_{1}^{u} \boldsymbol{e}_{1} e^{i \zeta_{1} x_{3}}+C_{2}^{u} \boldsymbol{e}_{2} e^{i \zeta_{2} x_{3}}+C_{3}^{u} \boldsymbol{e}_{3} e^{i \zeta_{3} x_{3}}+C_{1}^{d} \boldsymbol{e}_{4} e^{-i \zeta_{1} x_{3}}\right. \\
& \left.+C_{2}^{d} \boldsymbol{e}_{5} e^{-i \zeta_{2} x_{3}}+C_{3}^{d} \boldsymbol{e}_{6} e^{-i \zeta_{3} x_{3}}\right) e^{-i\left(\xi_{1} x_{1}+\xi_{2} x_{2}-\omega t\right)}
\end{aligned}
$$

In this equation, $C_{i}^{u}$ and $C_{i}^{d}$ are constants associated with upward and downward traveling waves, respectively. Through-thickness wavenumbers are represented as $\zeta_{i}$, whereas in-plane wavenumbers are represented as $\xi_{i}$. The eigenvectors for the displacement field correspond to the $\boldsymbol{e}_{j}$ variables.

Based on the assumption that each composite ply is transversely isotropic, the global matrix approach introduced by Lih and Mal [్] is used to account for the multilayer structure of the plate. A 2-D spatial and a temporal Fourier transform are used to represent the solution in the wavenumber and frequency domain. This facilitates the implementation of the differential operators required to formulate the stresses for the problem. Coordinate transformations are used to convert local in-plane wavenumbers to global in-plane wave numbers in polar coordinates. Subsequently, the global matrix is formed by enforcing displacement and stress continuity at the layer interfaces of the laminate. It is convenient to represent the stress and displacement quantities for a single lamina $m$ in vector form as

$$
\left[\begin{array}{cc}
\boldsymbol{L}^{m} & 0 \\
0 & \boldsymbol{L}^{m}
\end{array}\right]\left[\begin{array}{ll}
\boldsymbol{Q}_{11}^{m} & \boldsymbol{Q}_{12}^{m} \\
\boldsymbol{Q}_{21}^{m} & \boldsymbol{Q}_{22}^{m}
\end{array}\right]\left[\begin{array}{cc}
\boldsymbol{E}_{u}^{m} & 0 \\
0 & \boldsymbol{E}_{d}^{m}
\end{array}\right]\left[\begin{array}{l}
\boldsymbol{C}_{u}^{m} \\
\boldsymbol{C}_{d}^{m}
\end{array}\right]=\boldsymbol{Q}^{m} \boldsymbol{C}^{m}
$$

where $\boldsymbol{L}^{m}$ is the transformation matrix corresponding to the orientation of the $m$ th lamina. The $\boldsymbol{Q}_{i j}^{m}$ terms represent submatrices containing the stress and displacement eigenvectors in the local coordinate system. The contents of these submatrices can be found in detail in [ $[\underline{5}, 6]$. The terms of the $\boldsymbol{E}_{u}^{m}$ and $\boldsymbol{E}_{d}^{m}$ matrices are the exponentials from Eq. (2). The displacements and the three stress components contained in this vector are assumed to be continuous at the lamina interface between the bottom of lamina $m$ and the top of lamina $m+1$ :

$$
\boldsymbol{Q}_{\mathrm{Bottom}}^{m} \boldsymbol{C}^{m}=\boldsymbol{Q}_{\mathrm{Top}}^{m+1} \boldsymbol{C}^{m+1}
$$

and the interface between lamina $m$ and lamina $m+1$ can be represented as

$$
\left[\begin{array}{ll}
\boldsymbol{Q}_{\text {Bottom }}^{m} & -\boldsymbol{Q}_{\mathrm{Top}}^{m+1}
\end{array}\right]\left[\begin{array}{c}
\boldsymbol{C}^{m} \\
\boldsymbol{C}^{m+1}
\end{array}\right]=\left[\begin{array}{l}
0 \\
0
\end{array}\right]
$$

Figure 3 shows the layer-numbering scheme used in this formulation. Applying this relationship to each interface results in the formation of the banded global matrix, which completely represents the laminated plate structure. The global matrix will have size $6 N \times 6 N$, where $N$ is

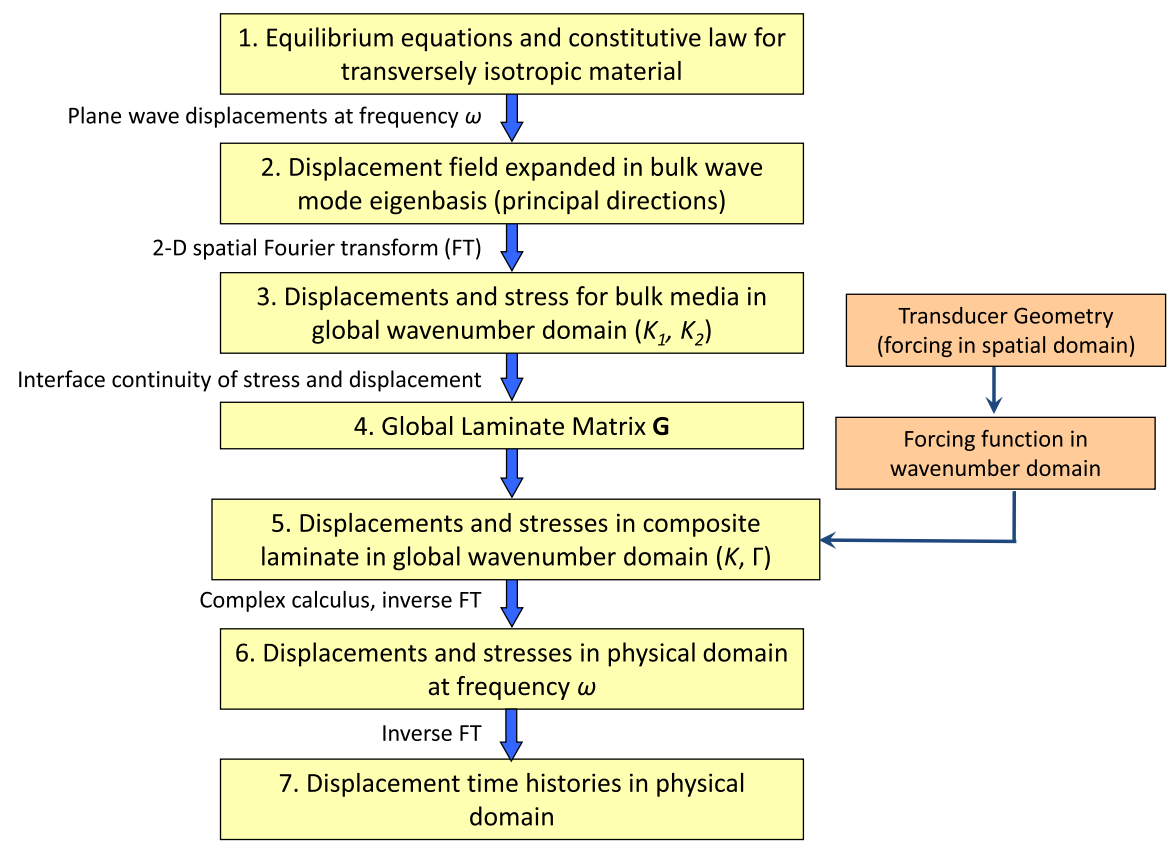

Fig. 2 Overview of global matrix method. 


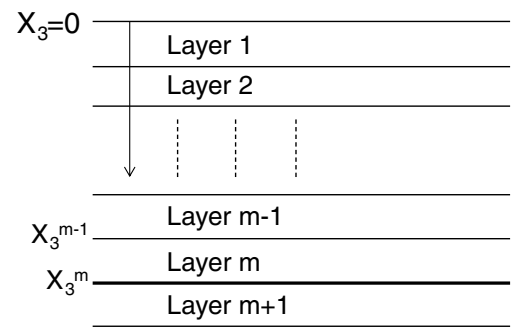

Fig. 3 Schematic of laminated plate structure.

the number of layers in the plate. When actuators are present on the top or bottom surface of the plate, these can be added to the forcing vector on the right-hand side of

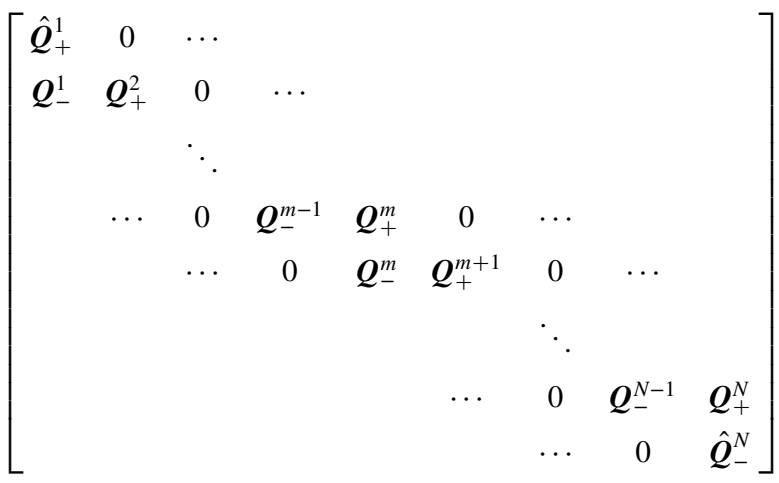

$$
\begin{aligned}
& \times\left[\begin{array}{c}
\boldsymbol{C}^{1} \\
\boldsymbol{C}^{2} \\
\vdots \\
\\
\\
\\
\\
\\
\\
\end{array}\right]=\left[\begin{array}{c}
\boldsymbol{F} \\
0 \\
\end{array}\right]
\end{aligned}
$$

Admissible in-plane wavenumbers are those that result in the determinant of the global matrix having a zero value. These wavenumbers can be used to calculate the admissible phase velocities and form dispersion curves for the plate. A complete solution of Eq. (6) is needed to determine the amplitude constants found in the displacement solution for the plate. This requires the forcing vector for the actuator to be represented in terms of a 2-D spatial Fourier transform. Any actuator can be modeled using this approach as long as the tractions it produces can be represented in the wavenumber domain. Cramer's rule provides a convenient way to determine the constant values once the forcing vector is in wavenumber space.

Having determined the constants for each lamina, it is now possible to determine the displacements at any point on the plate. As mentioned earlier, most previous implementations of this method have only allowed for determination of the out-of-plane displacement component at the top surface of the plate. The hybrid model described in this work requires that all three displacement components be specified at certain locations in the model. For this reason, previous implementations have been extended to calculate all three displacement components. Additionally, capability has been added to determine displacement components at all lamina interface locations, not just the plate surface. The displacement vector at a given point on the plate is represented in the wavenumber and frequency domain as

$$
\boldsymbol{U}\left(K_{1}, K_{2}, X_{3}, \omega\right)=\left[\begin{array}{ll}
\boldsymbol{Q}_{11}^{m} & \boldsymbol{Q}_{12}^{m}
\end{array}\right]\left[\begin{array}{cc}
\boldsymbol{E}_{u} & 0 \\
0 & \boldsymbol{E}_{d}
\end{array}\right]\left[\begin{array}{l}
\boldsymbol{C}_{u}^{m} \\
\boldsymbol{C}_{d}^{m}
\end{array}\right]
$$

where $X_{3}$ is the through-thickness location of the point of interest in global coordinates, $\omega$ is the frequency, and $\boldsymbol{Q}_{11}^{m}$ and $\boldsymbol{Q}_{12}^{m}$ are the displacement eigenvectors for the lamina containing the point of interest. The $\boldsymbol{C}^{m}$ constants used in the calculation also correspond the lamina containing the point of interest.

The previous matrix of exponentials must be calculated based on the through-thickness location of the point of interest. This matrix is in terms of the applicable lamina's local coordinate system and is given by

$$
\left[\begin{array}{cc}
\boldsymbol{E}_{u} & 0 \\
0 & \boldsymbol{E}_{d}
\end{array}\right]=\left[\begin{array}{cccccc}
e^{i \zeta_{1} x_{3}} & 0 & 0 & 0 & 0 & 0 \\
0 & e^{i \zeta_{2} x_{3}} & 0 & 0 & 0 & 0 \\
0 & 0 & e^{i \zeta_{3} x_{3}} & 0 & 0 & 0 \\
0 & 0 & 0 & e^{-i \zeta_{1} x_{3}} & 0 & 0 \\
0 & 0 & 0 & 0 & e^{-i \zeta_{2} x_{3}} & 0 \\
0 & 0 & 0 & 0 & 0 & e^{-i \zeta_{3} x_{3}}
\end{array}\right]
$$

Once the matrices in Eq. (7) are calculated, they can be multiplied to determine the value of the displacement vector at the given wavenumber, through-thickness location, and frequency. It can be shown that this results in an expression for displacement component $i$ of the form

$$
U_{i}\left(K_{1}, K_{2}, X_{3}, \omega\right)=\frac{\Psi_{i}\left(K, \Gamma, X_{3}, \omega\right)}{\Delta(K, \Gamma, \omega)}
$$

where the $\Delta$ term represents the determinant of the global matrix. The $\Psi_{i}$ term is a function of the azimuthal wavenumber and the constants associated with the layer of interest. The other displacement components are formed similarly. To determine the values of the displacement component in the spatial and time domains, a series of inverse transformations is necessary. First, a 2-D inverse Fourier transform is used to move from the wavenumber domain to the spatial domain. The inversion formula is given by

$$
u_{i}\left(r, \theta, X_{3}, \omega\right)=\frac{1}{4 \pi^{2}} \int_{0}^{\infty} \int_{0}^{2 \pi} \frac{\Psi_{i}\left(K, \Gamma, X_{3}, \omega\right)}{\Delta(K, \Gamma, \omega)} e^{-i K r \cos (\theta-\Gamma)} K \mathrm{~d} \Gamma \mathrm{d} K
$$

In Eq. (10), the integrand is singular at wavenumber values that make the determinant of the global matrix zero. These are exactly the values of the propagating wavenumbers for the plate. Previous works $[\underline{6}, \underline{7}, \underline{15}]$ have used complex calculus by implementing the residue theorem to perform the integration in the inversion formula. The result of the 2-D inversion is the displacement harmonic for the given spatial location and frequency. Finally, an inverse Fourier transform is used to move from the frequency domain to the time domain. The nature of the actuation must be considered during this step. In this work, a Hann-modulated toneburst signal with a given center frequency is used to excite the plate. An example with a $75 \mathrm{kHz}$ center frequency is shown in Fig. 4. To determine the frequency spectrum of the displacement at a given point, the Fourier transform of the actuation signal is multiplied by the displacement harmonic at each frequency. The inverse transform then produces the displacement time history at the point of interest.

\section{B. Actuator Modeling}

In this work, circular, square, and composite long-range variabledirection emitting radar (CLoVER) actuators are considered. For the circular actuator, the transformed forcing vector has previously [7] been shown to be

$$
F_{1}=-i \tau_{0} R J_{1}(K R) \cos \Gamma \quad F_{2}=-i \tau_{0} R J_{1}(K R) \sin \Gamma
$$

where $\tau_{0}$ is an amplitude constant for the stress transferred to the plate, $R$ is the radius of the actuator, $K$ is the global radial wavenumber, and $J_{1}$ represents the first-order Bessel function of the first kind. 

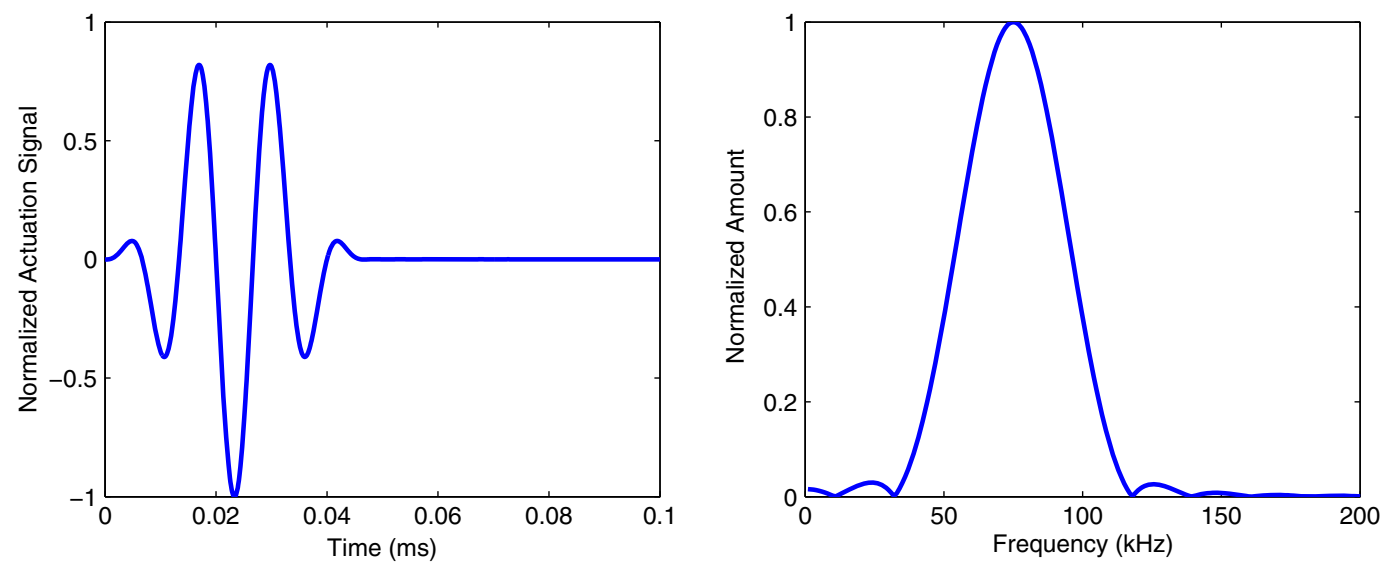

Fig. 4 Actuation signal time history and frequency content for a Hann-modulated toneburst with $75 \mathrm{kHz}$ center frequency.

For a rectangular actuator, the transformed forcing vector has been written [] $]$ as

$$
\begin{aligned}
& F_{1}=-4 \tau_{0} \sin \left(K_{1} A_{1}\right) \sin \left(K_{2} A_{2}\right) / i K_{2} \\
& F_{2}=-4 \tau_{0} \sin \left(K_{1} A_{1}\right) \sin \left(K_{2} A_{2}\right) / i K_{1}
\end{aligned}
$$

where $A_{1}$ and $A_{2}$ are half the lengths of the sides of the actuator. The $K_{1}$ and $K_{2}$ terms are the in-plane components of the global wavenumber.

The final actuator type considered in this study is the CLoVER. An example of it is shown in Fig. 5 . This actuator has not previously been incorporated into the global matrix method, and determination of the transformed forcing vector needed to accomplish this is a more lengthy process. However, a strategy for representing the tractions from a CLoVER sector was developed by Salas and Cesnik [16] to facilitate an exact isotropic solution, and that process can be adapted to produce the forcing terms needed for the global matrix equation. For a given CLoVER sector, the components of the forcing vector can be represented as

$$
\begin{aligned}
F_{1} & =\int_{0}^{2 \pi} \int_{0}^{\infty}\left[u\left(\theta-\theta_{L}\right)-u\left(\theta-\theta_{R}\right)\right]\left[\delta\left(r-R_{I}\right)\right. \\
& \left.-\delta\left(r-R_{O}\right)\right] \cos (\theta) e^{i K r \cos (\theta-\Gamma)} r \mathrm{~d} r \mathrm{~d} \theta \\
F_{2} & =\int_{0}^{2 \pi} \int_{0}^{\infty}-\left[u\left(\theta-\theta_{L}\right)-u\left(\theta-\theta_{R}\right)\right]\left[\delta\left(r-R_{I}\right)\right. \\
& \left.-\delta\left(r-R_{O}\right)\right] \sin (\theta) e^{i K r \cos (\theta-\Gamma)} r \mathrm{~d} r \mathrm{~d} \theta
\end{aligned}
$$

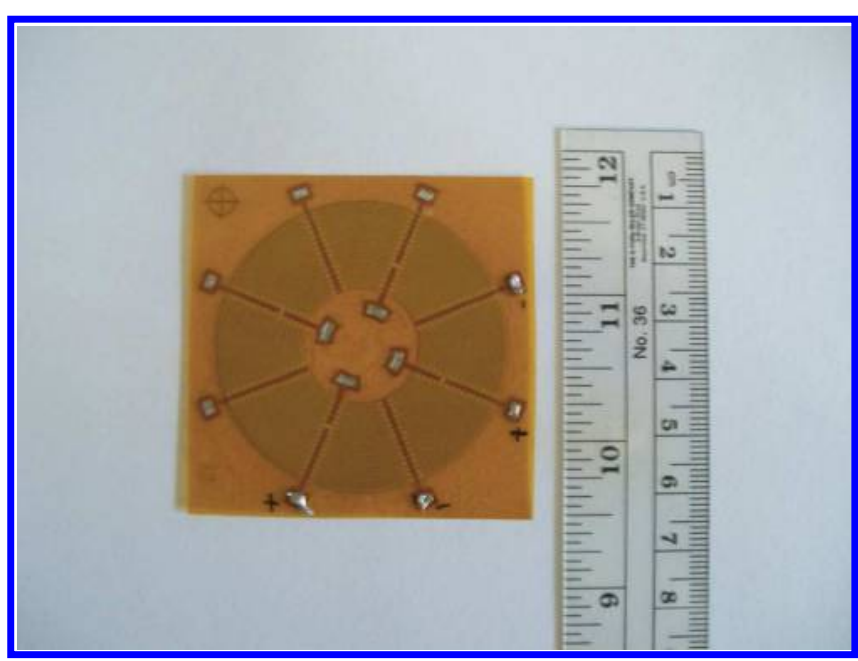

Fig. 5 CLoVER actuator. where $\theta_{L}, \theta_{R}$ represent the azimuthal boundaries of the sector of interest, and $R_{I}, R_{O}$ are its radial boundaries. The integrals in Eq. (13) cannot be solved analytically, but it is possible to use a Fourier series representation of the forcing terms to circumvent this difficulty:

$$
\begin{aligned}
F_{j} & =\sum_{M=-\infty}^{\infty} c_{M}^{(j)} e^{i M \Gamma}(-i)^{M} 2 \pi\left[R_{O} J_{M}\left(K R_{O}\right)-R_{I} J_{M}\left(K R_{I}\right)\right] \\
j & =1,2
\end{aligned}
$$

In Eq. (14), the $c_{M}$ terms are Fourier coefficients that must be calculated for each direction and each term of the series. The formulas for these coefficients can be found in [16]. In that work, it is also noted that satisfactory results can be obtained while only including the first 150 positive and negative terms of the Fourier series.

\section{Local Interaction Simulation Approach}

Although the global matrix method can accurately capture wave propagation for a pristine plate with no lateral boundaries, numerical approaches are needed to capture features present in realistic structures. Modeling wave propagation using the finite element method has been a popular technique, but it is often inefficient due to the computational cost involved in resolving short wavelengths with small time increments. Delsanto et al. [10-12] introduced an attractive alternative: LISA. This method is based on iterative equations (IEs) for unit cells that are used to discretize the model. The actual IEs are derived from the elastodynamic equilibrium equations [14] in Eq. (1). finite difference approximations form the backbone of the derivation, which can be seen in detail in several previous papers $[8,12,17]$. Figure 6 summarizes the typical process of deriving the IE for LISA.

The coefficients in the LISA IE depend only on the local physical properties. LISA uses a sharp interface model where the actual conditions enforced at the cell interfaces are continuity of displacements and stresses. Therefore, changes in stiffness, density, or attenuation can be accounted for because the IE coefficients in adjacent cells with different properties will vary. The resulting formulation determines the response of any point at the current time step based on the response of that point's 18 nearest neighbors (shown in Fig. 7) in previous time steps.

Lee and Staszewski $[9,13,18,19]$ showed the utility of LISA for isotropic plates and extended their work to model simple damage in those plates using a 2-D model. Sundararaman and Adams used a 3-D LISA formulation to study the effect of simple damage in isotropic and unidirectional orthotropic plates [8] and subsequently examined the effects of grid size [20]. Recently, Nadella and Cesnik [14,21] extended the approach to simulate wave propagation in arbitrary composite laminates and expanded the 3-D formulation to account for plies in nonprincipal directions. The resulting IE are used in this work, and they are included for reference in the Appendix. Their work has previously demonstrated LISA's usefulness in modeling a 


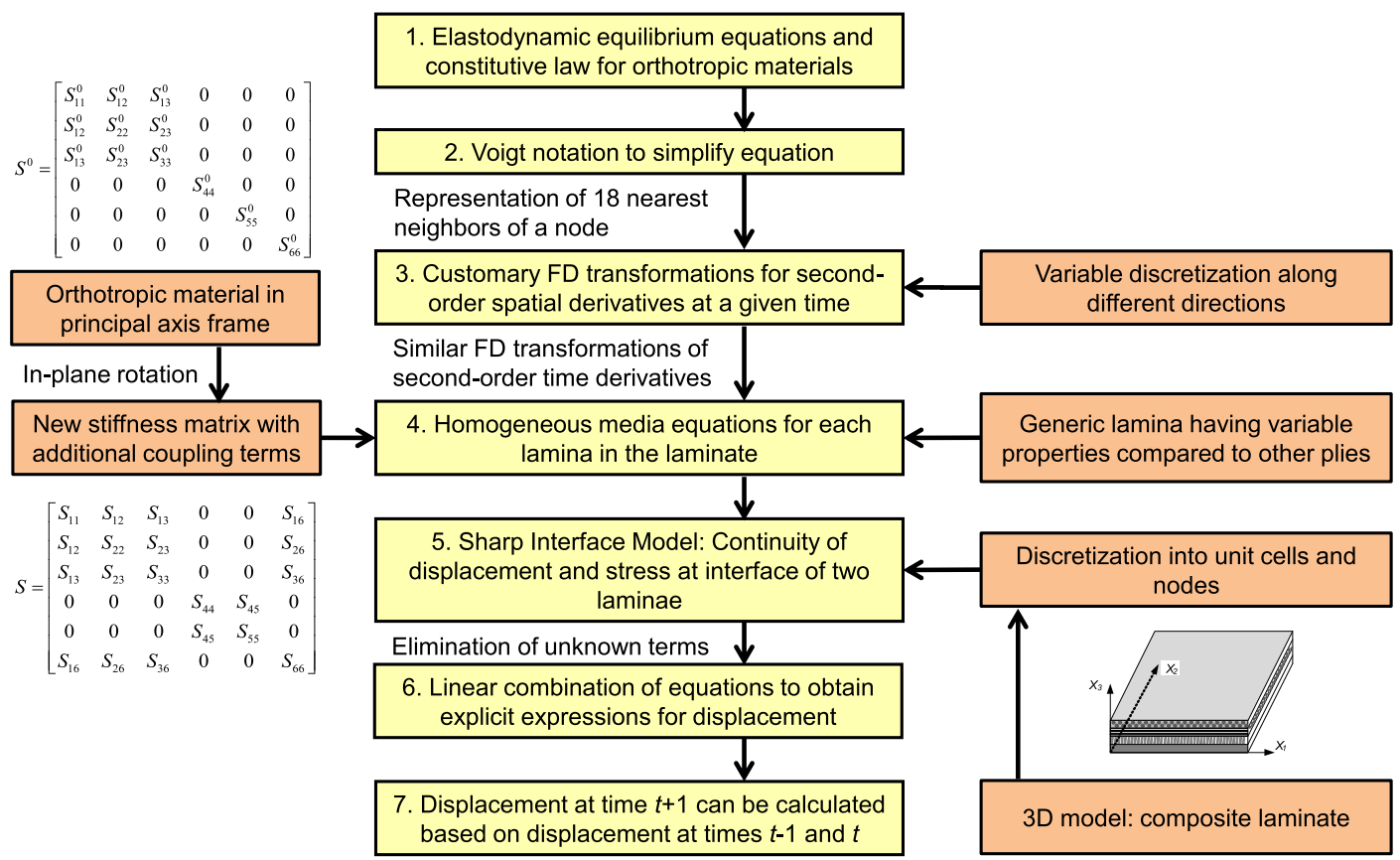

Fig. 6 Overview of LISA theoretical derivation.

variety of composite configurations including unidirectional, crossply, and quasi-isotropic laminates.

In most previous studies, actuation was modeled by prescribing inplane displacements at surface nodes located within the profile of the actuator. Schematics of this actuation method for circular lead zirconium titanate (PZT) and CLoVER actuators are shown in Figs. 8 and 9. Two major difficulties have emerged with actuator modeling of this type. First, as seen in the schematic, the Cartesian grid associated with current LISA implementations does not lend itself well to capturing the nonlinear boundaries of circular actuators or more sophisticated actuators such as CLoVER. In many circumstances, the boundaries of the actuator are located between nodes, and some judgment must be exercised to determine which nodes to include in the actuator profile. The second difficulty is the need to assume values

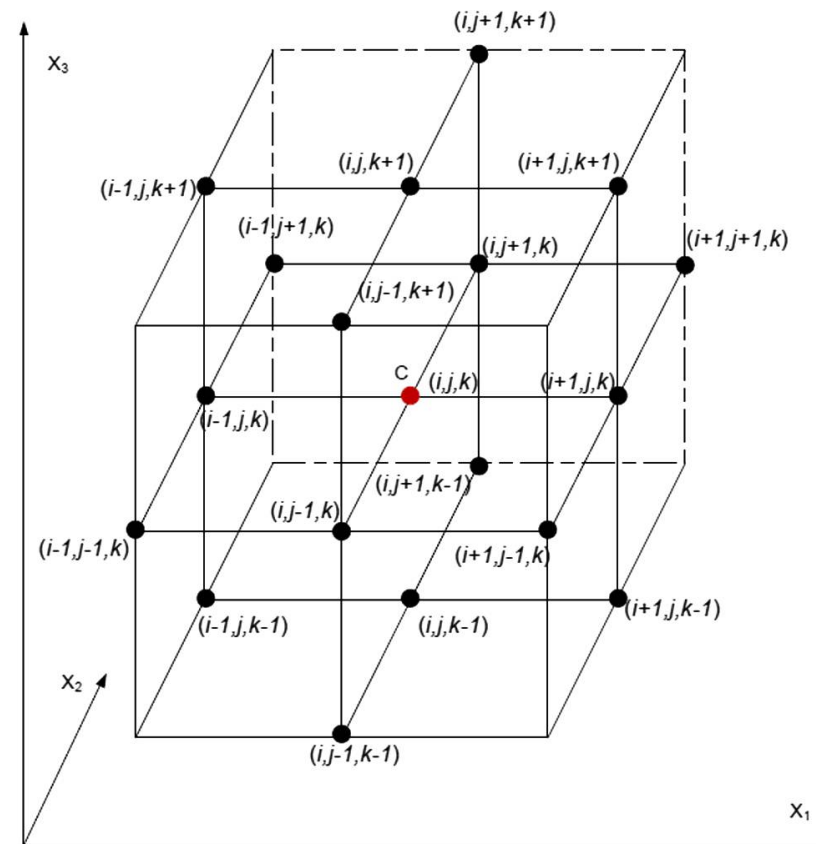

Fig. 7 Representation of the 18 neighbors of a generic point in LISA and the Cartesian system used in the analysis [14]. for the displacements applied at the nodes contained in the actuator profile. For example, the scheme illustrated for the circular actuator shows a linear increase in the radial displacement based on the radial distance from the actuator center, but that assumption may not truly reflect the displacements created by the actuator, especially for anisotropic materials. The LISA hybrid model presented in this paper avoids both of these difficulties. In the next section, the effectiveness
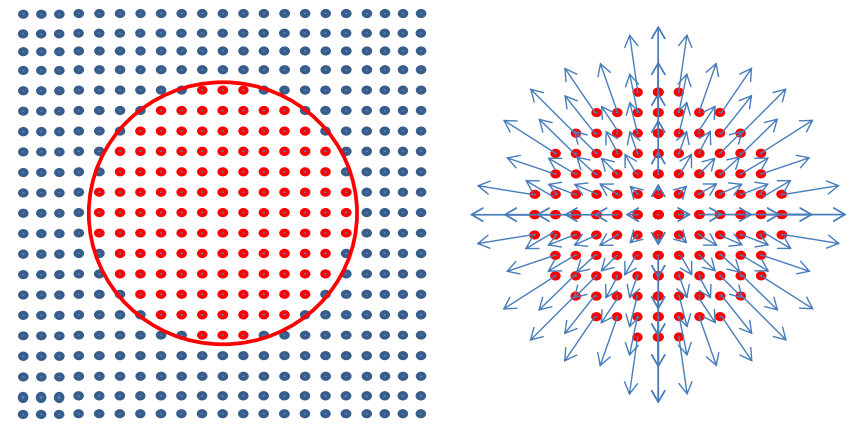

Fig. 8 Original LISA actuation scheme for a circular actuator using specified in-plane surface displacements.

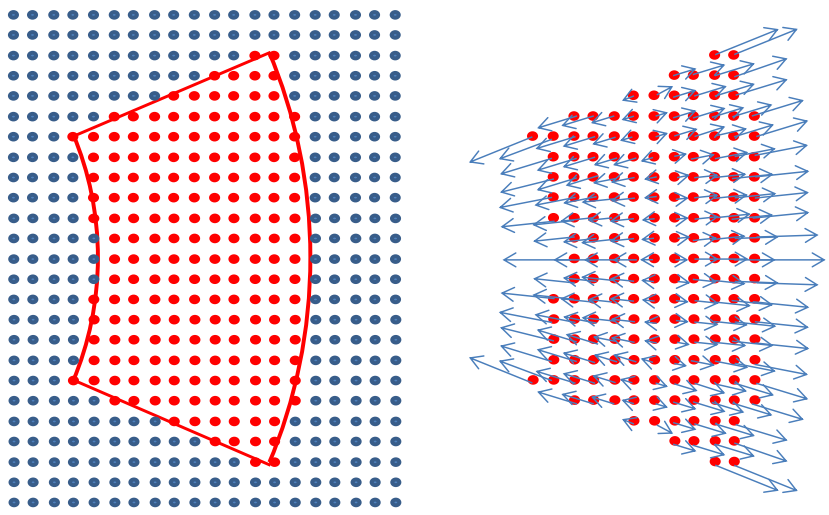

Fig. 9 Original LISA actuation scheme for a CLoVER actuator sector using specified in-plane surface displacements. 
Table 1 Graphite-epoxy lamina material properties

\begin{tabular}{lccccccc}
\hline \hline Property & $E_{1}$ & $E_{2}$ & $G_{12}$ & $G_{23}$ & $\nu_{12}$ & $\rho$ & $t_{p l v}$ \\
\hline Value & $147 \mathrm{GPa}$ & $9.8 \mathrm{GPa}$ & $2.35 \mathrm{GPa}$ & $3.3 \mathrm{GPa}$ & 0.405 & $1558 \mathrm{~kg} / \mathrm{m}^{3}$ & $0.125 \mathrm{~mm}$ \\
\hline \hline
\end{tabular}

Table 2 Aluminum material properties

\begin{tabular}{lcccc}
\hline \hline Property & $E$ & $\nu$ & $\rho$ & $t$ \\
\hline Value & $70 \mathrm{GPa}$ & 0.3 & $2700 \mathrm{~kg} / \mathrm{m}^{3}$ & $1.5 \mathrm{~mm}$ \\
\hline \hline
\end{tabular}

of both the previous approach and the LISA hybrid model is examined with respect to theoretical results.

\section{Results and Discussion}

To evaluate the effectiveness of the LISA hybrid model in comparison with the previous LISA implementations, a series of models were evaluated for an aluminum plate, a cross-ply plate $\left([0 / 90]_{3 S}\right)$, a unidirectional plate $\left([0]_{12 T}\right)$, and a quasi-isotropic plate $\left([0 / 30 / 60 /-60 /-30 / 90]_{S}\right)$. Each plate had a thickness of $1.5 \mathrm{~mm}$, and the composite plates contained 12 layers of the graphiteepoxy lamina from Table 1 . Material properties for the aluminum plate are shown in Table 2 .

In most of the LISA models, a cell size of $0.25 \times 0.25 \times 0.125 \mathrm{~mm}$ was used along with a time step of $10 \mathrm{~ns}$. These parameters meet the Courant-Friedrichs-Lewy (CFL) criterion,

$$
\mathrm{CFL}=c_{\max } \Delta t \sqrt{\frac{1}{\Delta x_{1}^{2}}+\frac{1}{\Delta x_{2}^{2}}+\frac{1}{\Delta x_{3}^{2}}} \leq 1
$$

which is a necessary condition for stability for FD-based numerical schemes [8]. As defined in Eq. (15), the CFL number must remain less than 1, and it is dependent on the time step used in the model as well as the spatial discretization. The $c_{\max }$ term represents the maximum propagating wave speed. A brief discretization study was also performed using coarser in-plane cell sizes, which also meet the CFL criterion.

In this section, results for circular, CLoVER, and square actuators will be presented. The actuation was specified as a Hann-modulated toneburst with a $75 \mathrm{kHz}$ center frequency, as shown in Fig. 4 . Traction-free boundary conditions were implemented using a layer of air cells surrounding the plate.

The initial set of LISA models for each actuator type used the previous method of prescribed displacements to model the actuator, and these results are henceforth referred to as the LISA original model. Subsequently, each actuator was modeled using the LISA hybrid approach. The cutout region was defined as a 20 by $20 \mathrm{~mm}$ region for the circular and square actuator models and as a 60 by
$60 \mathrm{~mm}$ region for the CLoVER models. All models were compared against the results from the global matrix method because that approach was shown to produce results that agree well with experiments [6]. Because the displacements on the boundary of the cutout were specified as the global matrix displacements, the hybrid model exactly matched the global matrix results for all points located on this boundary. If the LISA hybrid model was successful in capturing the propagating wave, then the model results also matched the global matrix results at locations farther away from the actuator.

\section{A. Circular Actuator Case}

The first group of simulations considered a 6.4-mm-radius circular actuator on the previously identified plates. For the aluminum plate, the time histories predicted at three different radial distances from the center of the actuator are shown for the two LISA models as well as the global matrix method in Fig. 10. At each of the three locations, it can be seen that the LISA original results showed some discrepancies from the baseline global matrix results, but for the most part the results matched reasonably well, particularly at the $80 \mathrm{~mm}$ distance. The duration of the pulse in the LISA original model did seem to be shorter than that in the global matrix results, as indicated by the Hilbert transform envelope included in the figures. The LISA hybrid model, in contrast, appeared to match the global matrix results almost perfectly at each of the three distances shown.

The results from the models considering the circular actuator on the three composite plates are shown in Figs. 11-13.

Each of the time histories is for a point $40 \overline{\mathrm{mm}}$ from the actuator center. Results at 0,45 , and $90 \mathrm{deg}$ are presented for each plate because the results are not axisymmetric like in the aluminum case. The ability of the LISA original model to capture the wave propagation time histories was much worse for these models where the group velocities and attenuation varied with azimuthal direction. For each of the three plates, results at the three angles showed that the LISA original model did not closely match the baseline global matrix time history. Although the arrival times of the wave packets were roughly the same for all three models, the LISA original model showed discrepancies in the duration, amplitude, and shape of the wave packet in comparison to the baseline model. The LISA hybrid model again produced time histories matching closely with the global matrix results.

\section{Local Interaction Simulation Approach Hybrid Displacement Field}

The ability of the LISA hybrid model to capture the wave propagation time histories for a circular actuator at various points has been demonstrated. To better see the effectiveness of the LISA hybrid model in capturing the directional dependence of the wave
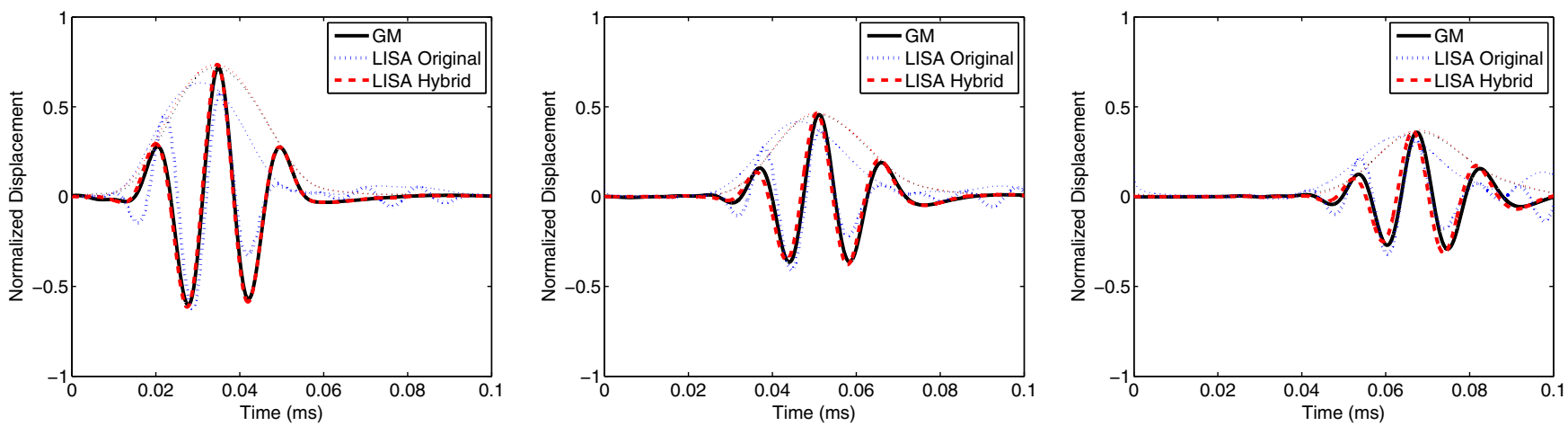

Fig. 10 Time history comparison for an aluminum plate. Locations 20,50 , and $80 \mathrm{~mm}$ from the circular actuator center are shown from left to right. 

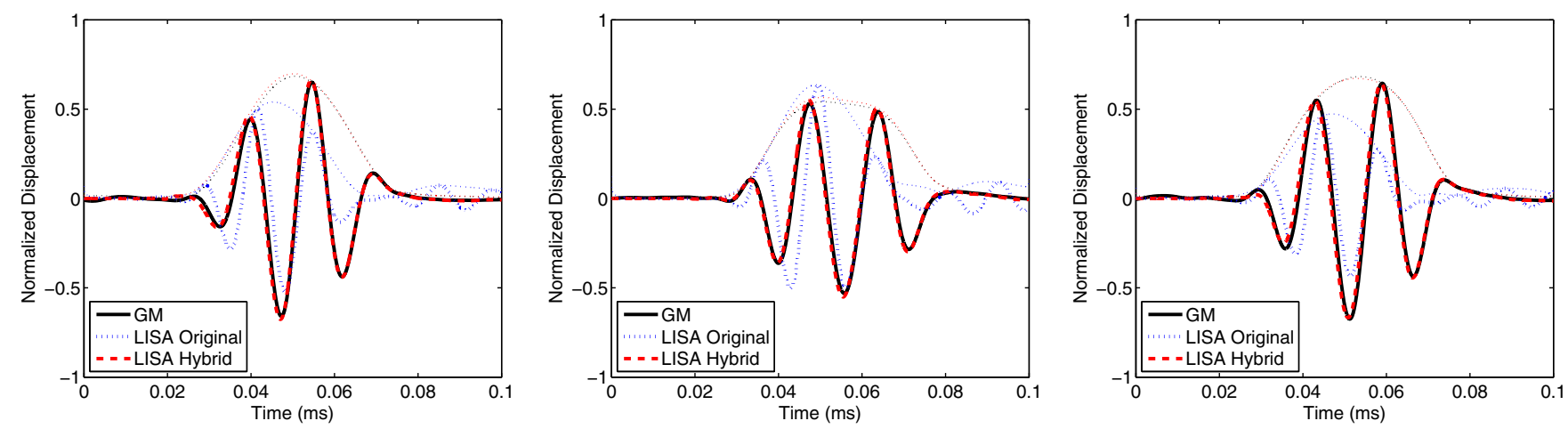

Fig. 11 Time history comparison for a cross-ply plate, $40 \mathrm{~mm}$ from the circular actuator center. The 0,45 , and 90 deg directions are shown from left to right.
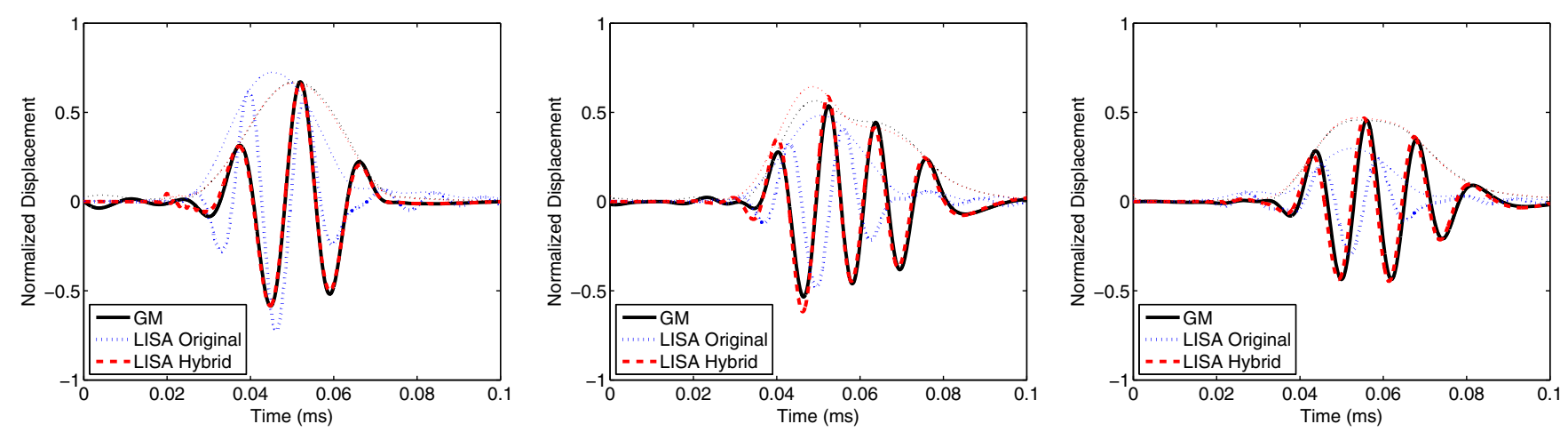

Fig. 12 Time history comparison for a unidirectional plate, $40 \mathrm{~mm}$ from the circular actuator center. The 0,45 , and 90 deg directions are shown from left to right.
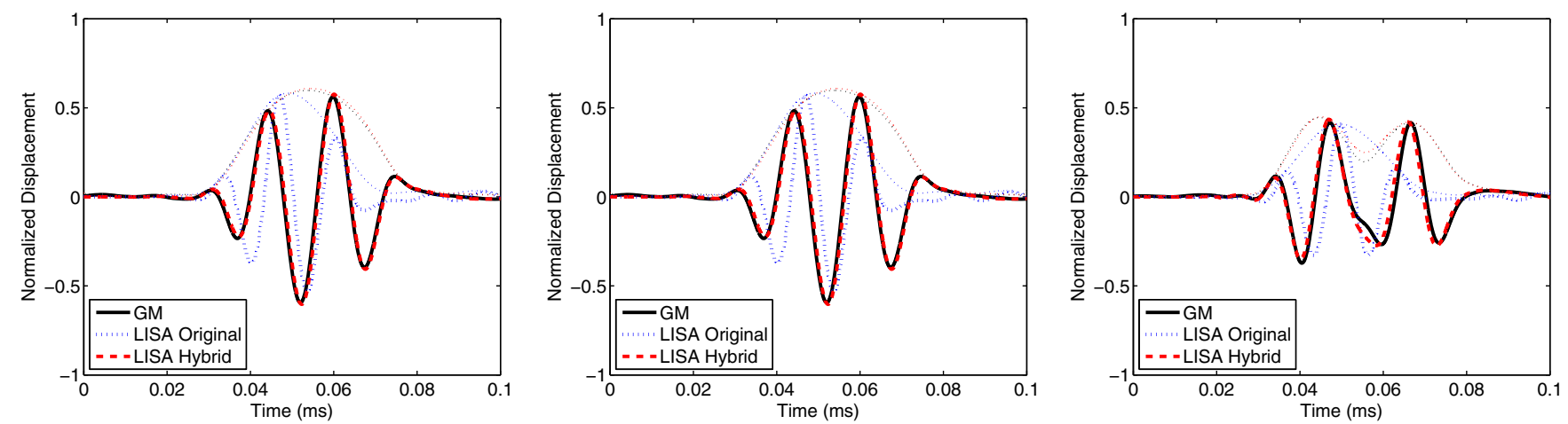

Fig. 13 Time history comparison for a quasi-isotropic plate, $40 \mathrm{~mm}$ from the circular actuator center. The 0,45 , and 90 deg directions are shown from left to right.

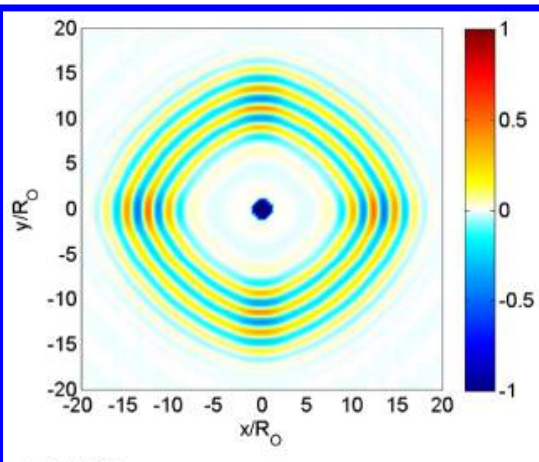

a) GM80 $\mu \mathrm{s}$

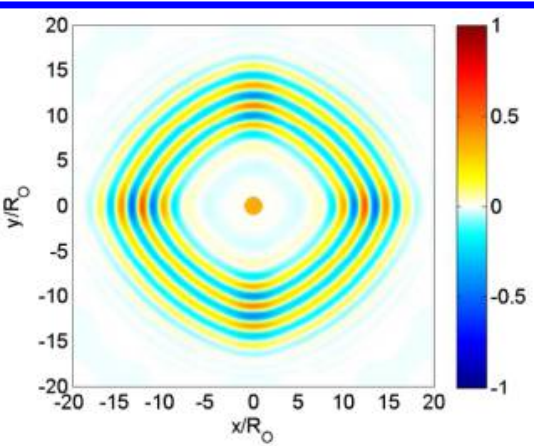

b) LISA hybrid $80 \mu \mathrm{s}$

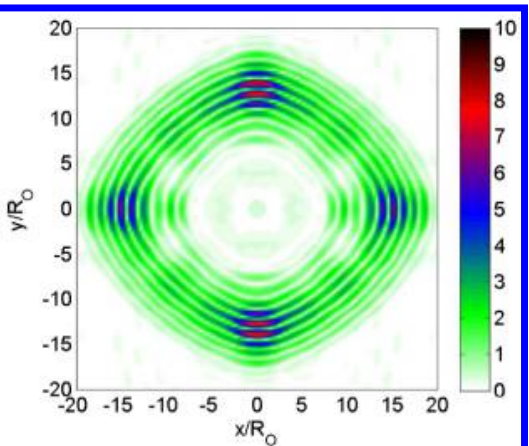

c) Error as \% of max amplitude

Fig. 14 Comparison of global matrix and LISA hybrid results for out-of-plane displacement of a cross-ply plate with a circular actuator at $80 \mu \mathrm{s}$ 

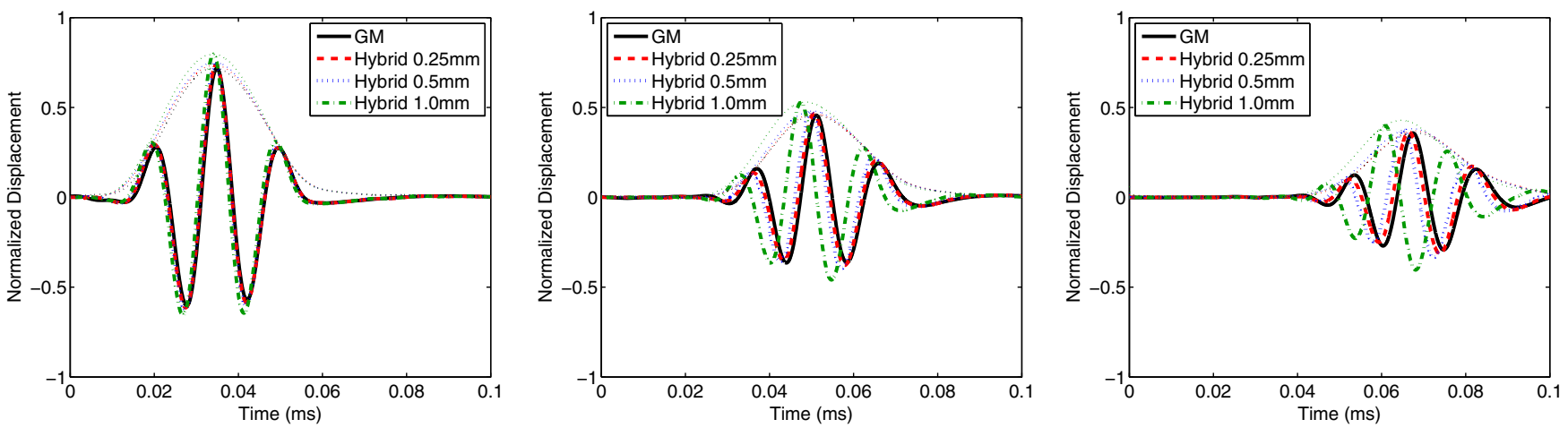

Fig. 15 Comparison of LISA Hybrid discretizations for an aluminum plate. Locations 20, 50, and $80 \mathrm{~mm}$ from the actuator center are shown from left to right.

propagation in a composite plate, Fig. 14 shows field plots of the outof-plane displacement of the cross-ply plate. The global matrix and LISA hybrid results are compared at $80 \mu \mathrm{s}$. The preponderance of energy propagating in the 0 and 90 deg directions was preserved well in the new modeling approach. Figure $14 \mathrm{c}$ shows the amplitude error present at each point on the plate as a percentage of the maximum amplitude in the propagating wave. The error was consistenly less than $10 \%$ of the maximum amplitude.

\section{Effect of In-Plane Discretization}

Previous researchers [20] have noted the dependence of LISA results on the in-plane discretization. This dependence was also seen in the present LISA hybrid results, particularly in the group velocity of the propagating wave. The four circular actuator models were run with in-plane cell sizes of $1.0,0.5$, and $0.25 \mathrm{~mm}$. The throughthickness discretization was held constant at $0.125 \mathrm{~mm}$. The results indicated the group velocity predicted by the LISA hybrid model increased as the in-plane cell size increased. This trend was very noticeable for the aluminum plate. As shown in Fig. 15, the results for each of the three discretization sizes agreed very will with the baseline model at $20 \mathrm{~mm}$. However, at more distant points, the effect of the group velocity differences was more apparent. The plot for the $80 \mathrm{~mm}$ location demonstrates the effect of overly coarse discretizations on the model results.

This effect was also seen in the composite models, and not surprisingly the effect was direction-dependent. The three discretization sizes were compared for the 0,45 , and $90 \mathrm{deg}$ directions at points $70 \mathrm{~mm}$ from the actuator center. At this distance, the group velocity differences had time to manifest themselves. Compared to the aluminum model, the cross-ply model shown in Fig. 16 and the quasiisotropic model shown in Fig. 17 were much less sensitive to the cell size, but the results were affected more for the 90 deg direction than
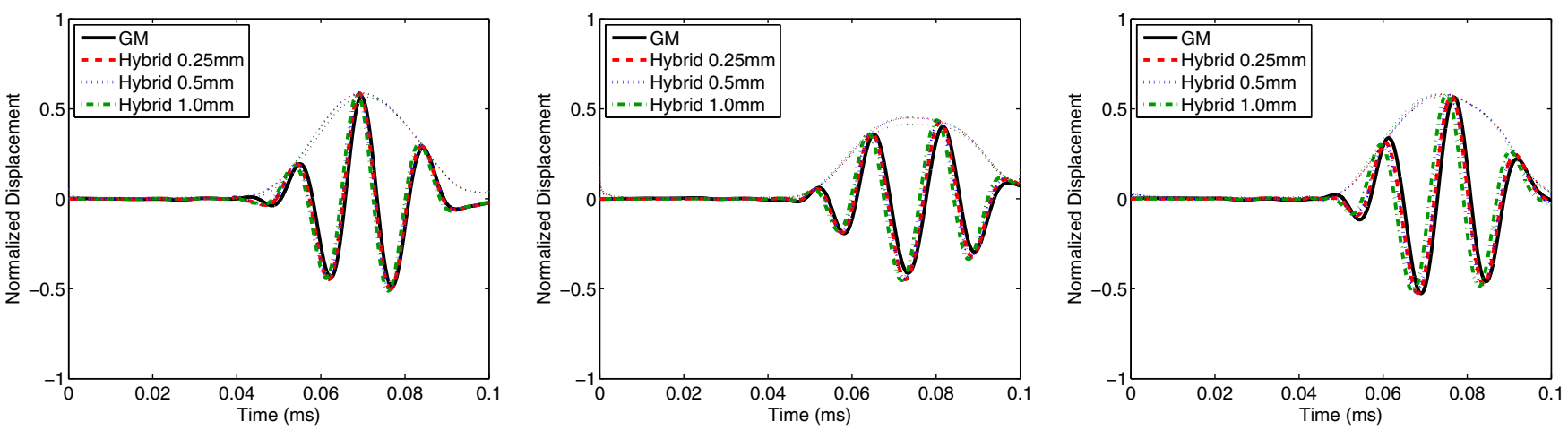

Fig. 16 Comparison of LISA hybrid discretizations for the cross-ply plate $70 \mathrm{~mm}$ from the actuator center. The 0, 45, and 90 deg directions are shown from left to right.
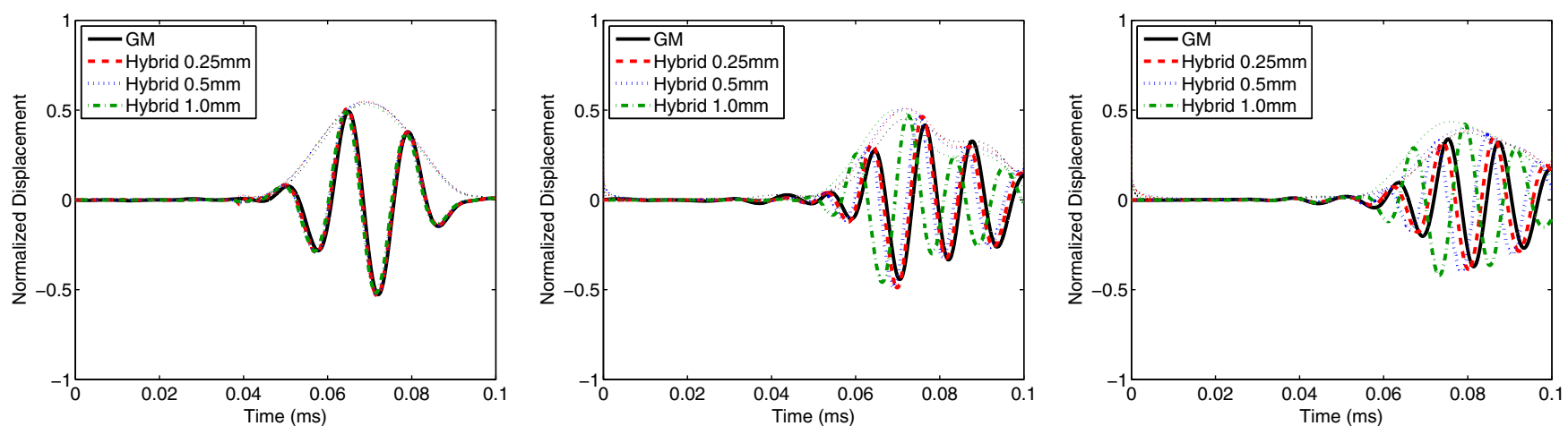

Fig. 17 Comparison of LISA hybrid discretizations for the quasi-isotropic plate $70 \mathrm{~mm}$ from the actuator center. The 0,45 , and 90 deg directions are shown from left to right. 

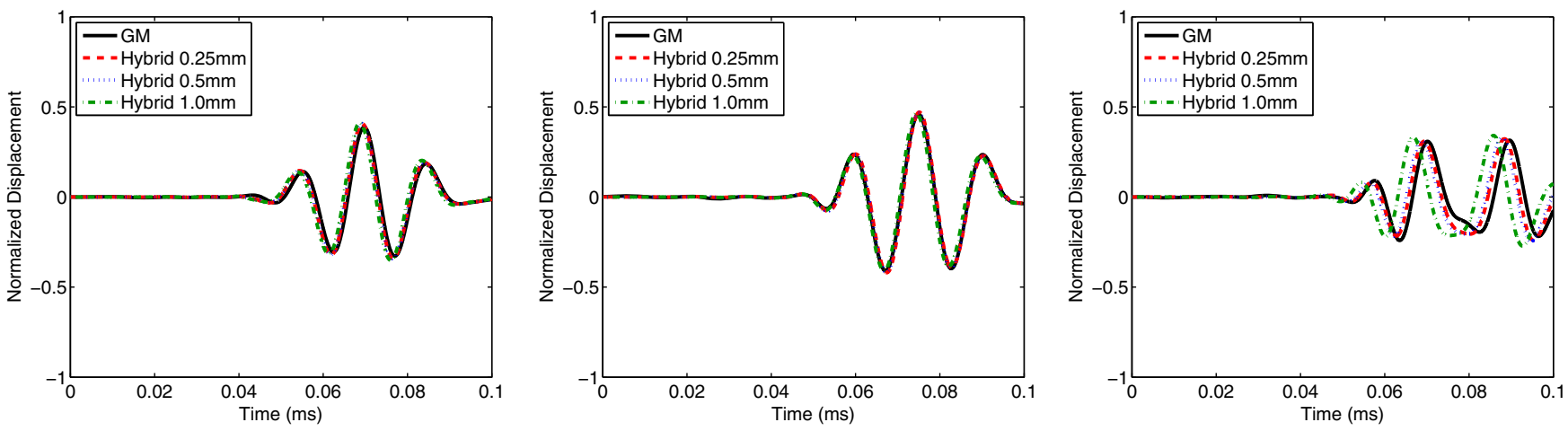

Fig. 18 Comparison of LISA hybrid discretizations for the unidirectional plate $70 \mathrm{~mm}$ from the actuator center. The 0,45 , and 90 deg directions are shown from left to right.
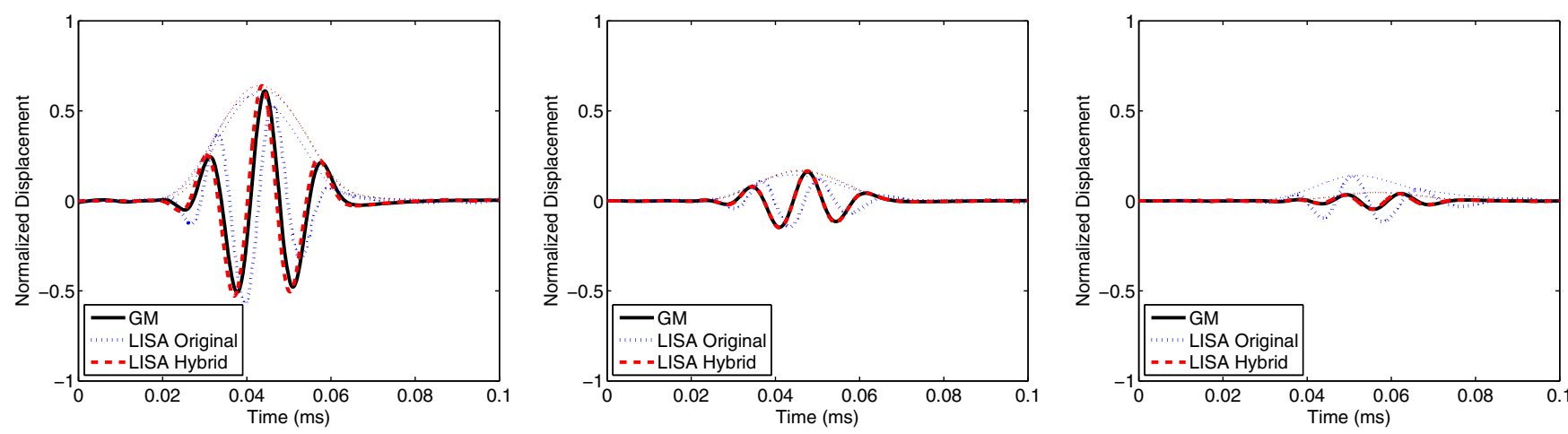

Fig. 19 Time history comparison for an aluminum plate with a 0 deg CLoVER sector active. The 0,45 , and 90 deg directions are shown from left to right.
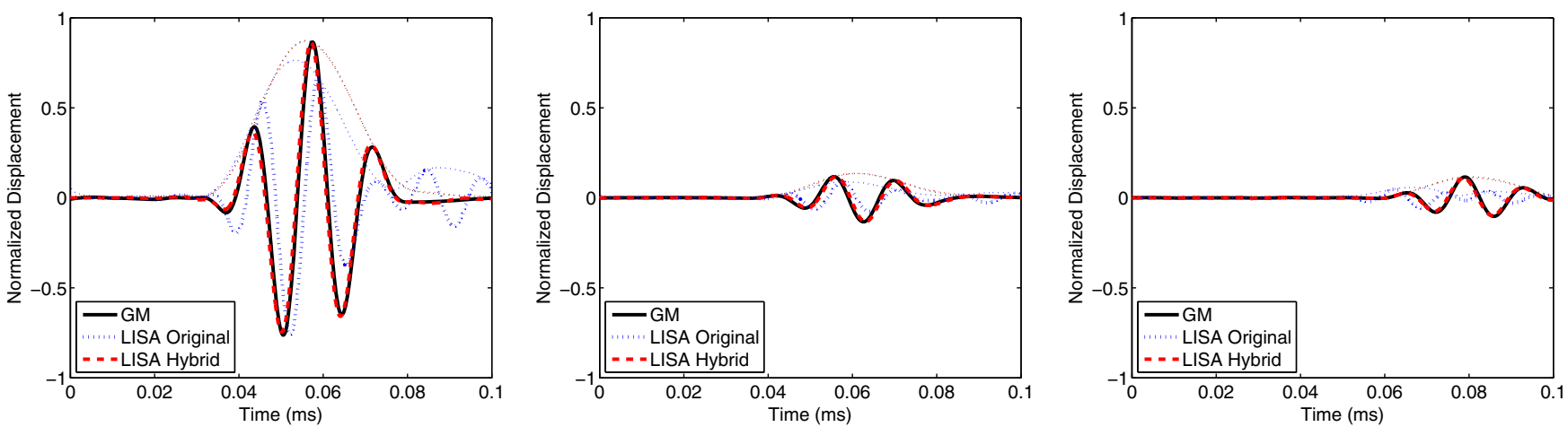

Fig. 20 Time history comparison for a cross-ply plate with a 0 deg CLoVER sector active. The 0,45 , and 90 deg directions are shown from left to right.
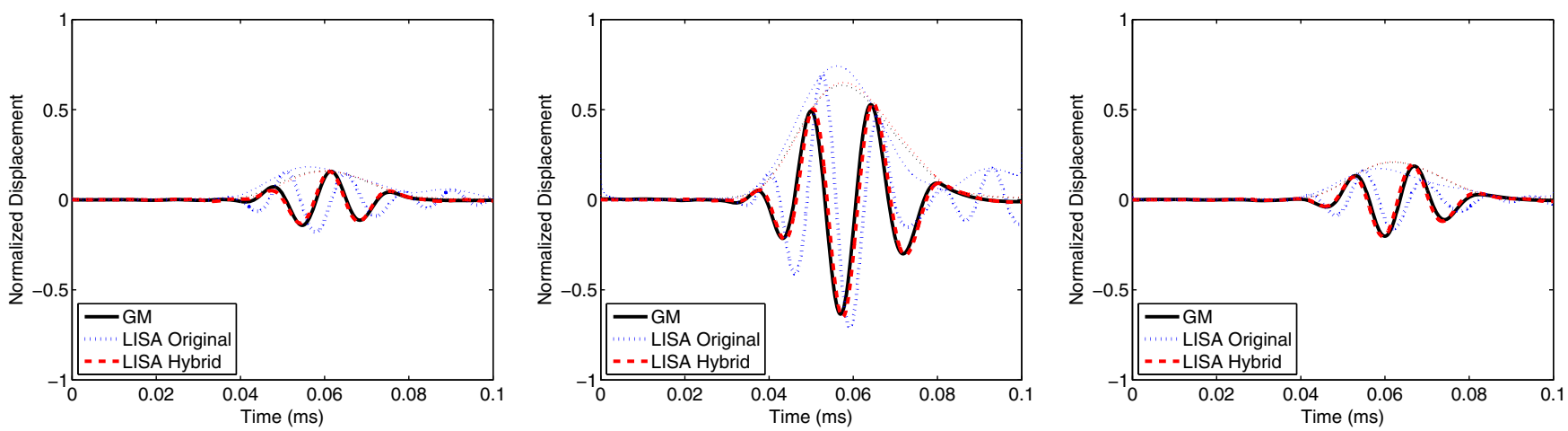

Fig. 21 Time history comparison for a cross-ply plate with a $45 \mathrm{deg}$ CLoVER sector active. The 0,45 , and 90 deg directions are shown from left to right. 

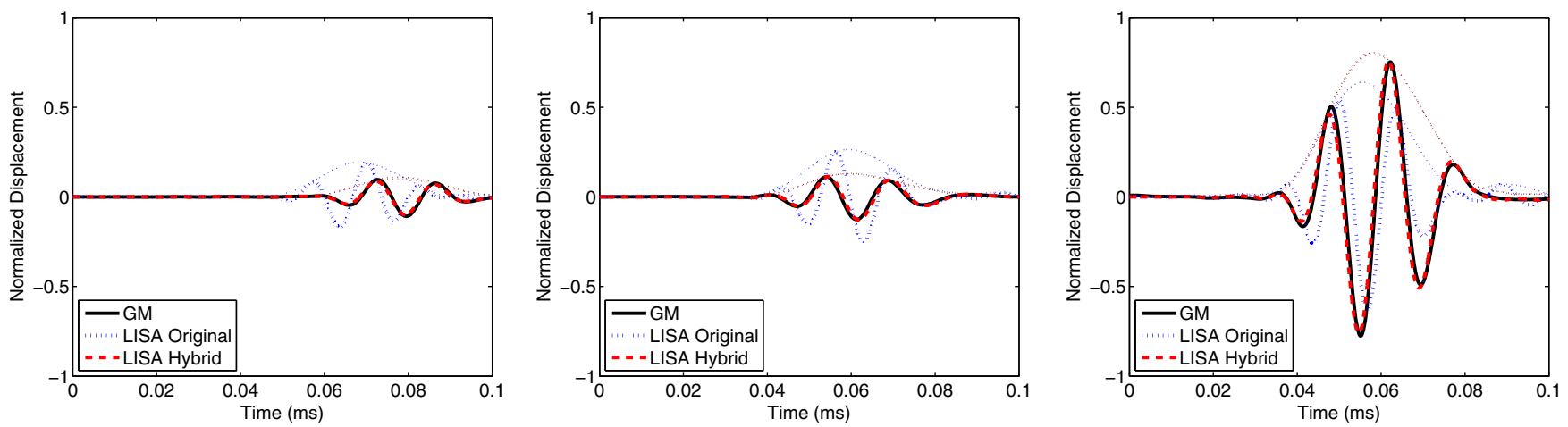

Fig. 22 Time history comparison for a cross-ply plate with a 90 deg CLoVER sector active. The 0,45 , and 90 deg directions are shown from left to right.

the 0 deg direction. For the unidirectional model, the directional dependence was even more pronounced, as seen in Fig. 18. Here, all three cell sizes produced good results at $0 \mathrm{deg}$, but the results for coarse discretizations worsened as the azimuth increased from 0 to $90 \mathrm{deg}$.
In the LISA hybrid model, as in previous LISA implementations, the group velocity was clearly a function of not only the material properties of the structure and the azimuthal direction but also the discretization used in the model. Errors in the group velocity due to the chosen discretization resulted in errors in the arrival time of the

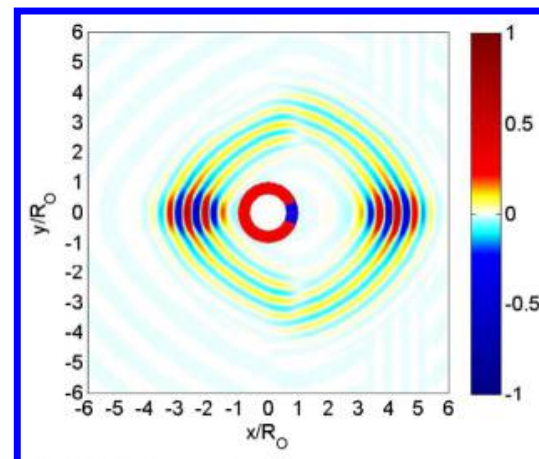

a) GM 0-deg sector

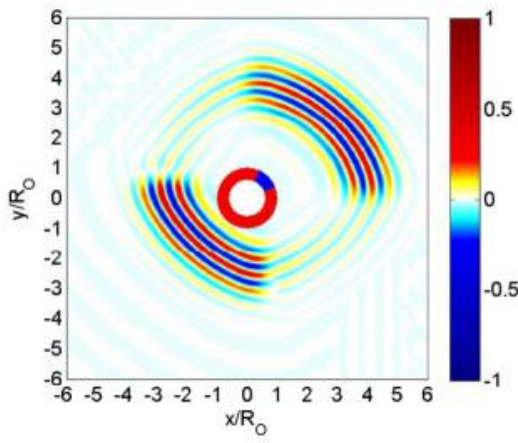

d) GM 45-deg sector

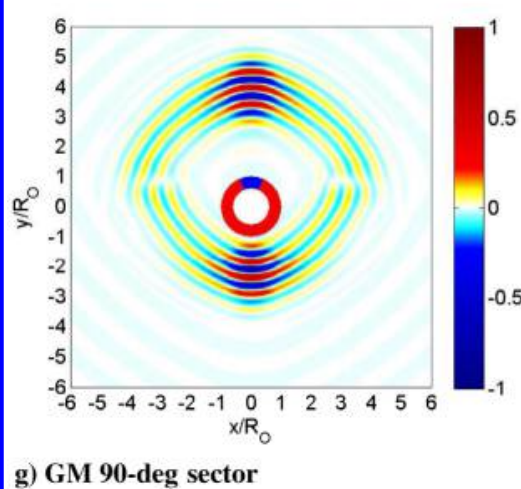

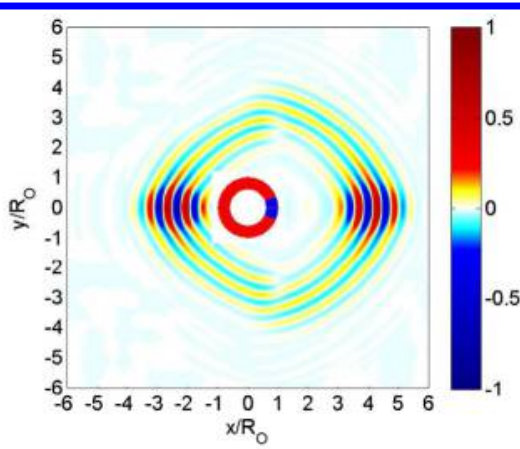

b) LISA hybrid 0-deg sector

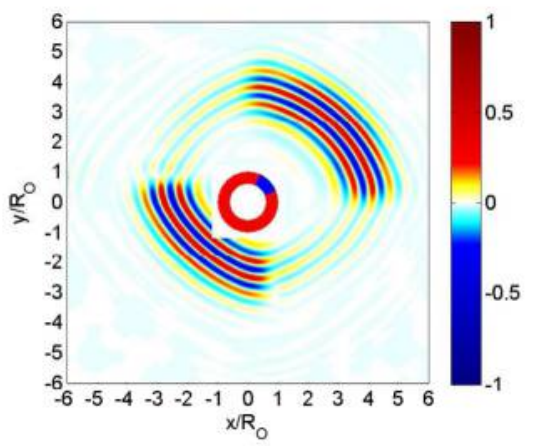

e) LISA hybrid 45-deg sector

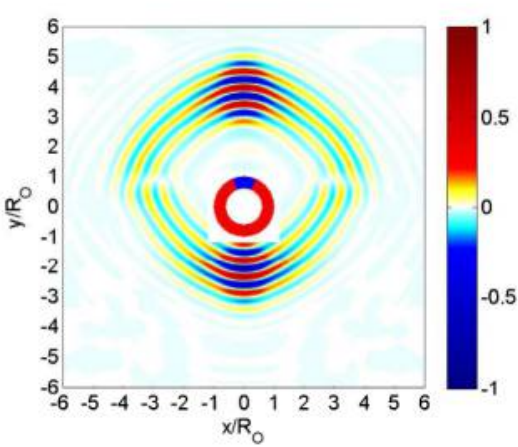

h) LISA hybrid 90-deg sector

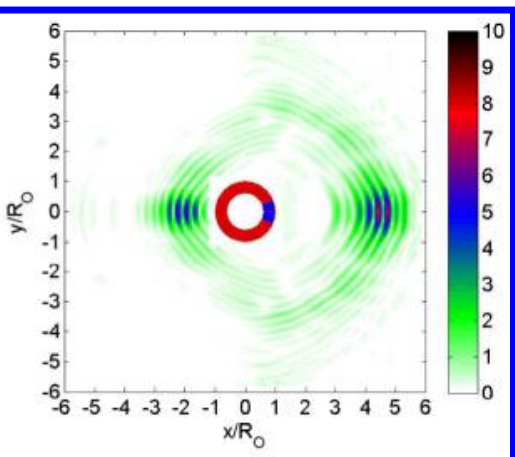

c) Error as \% of max amplitude

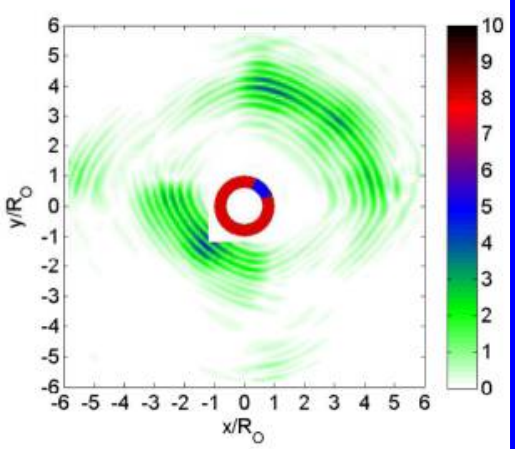

f) Error as \% of max amplitude

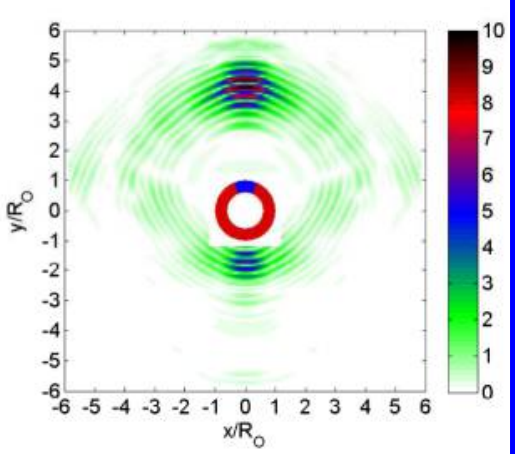

i) Error as \% of max amplitude

Fig. 23 Comparison of global matrix and LISA hybrid results for out-of-plane displacement of a cross-ply plate at $80 \mu \mathrm{s}$ with various active CLoVER sectors. 

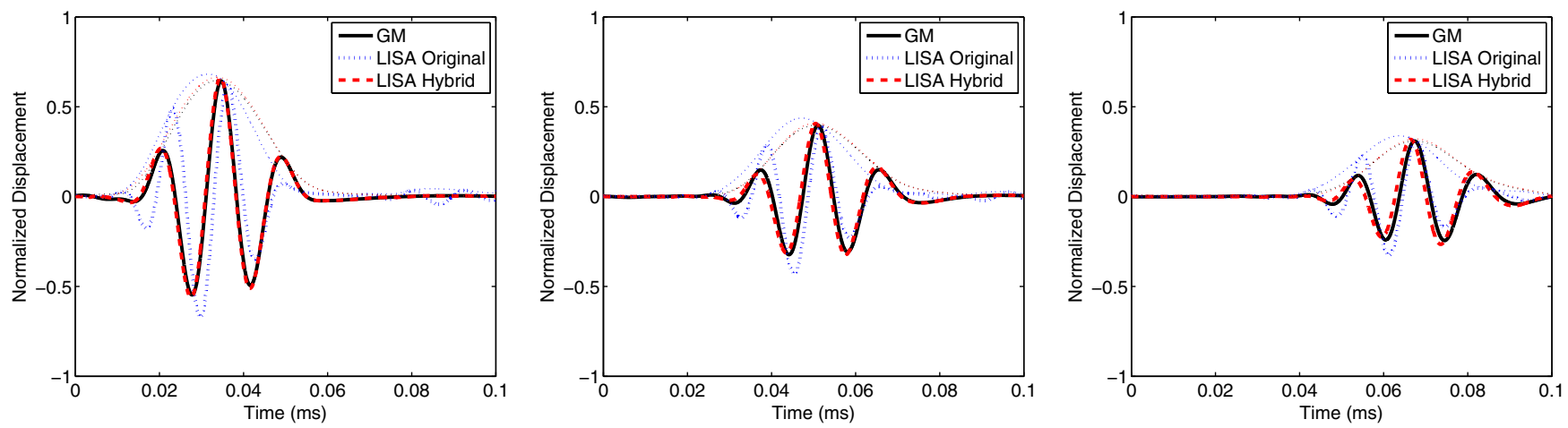

Fig. 24 Time history comparison for an aluminum plate. Locations $\mathbf{2 0}, \mathbf{5 0}$, and $80 \mathrm{~mm}$ from the square actuator center are shown from left to right.
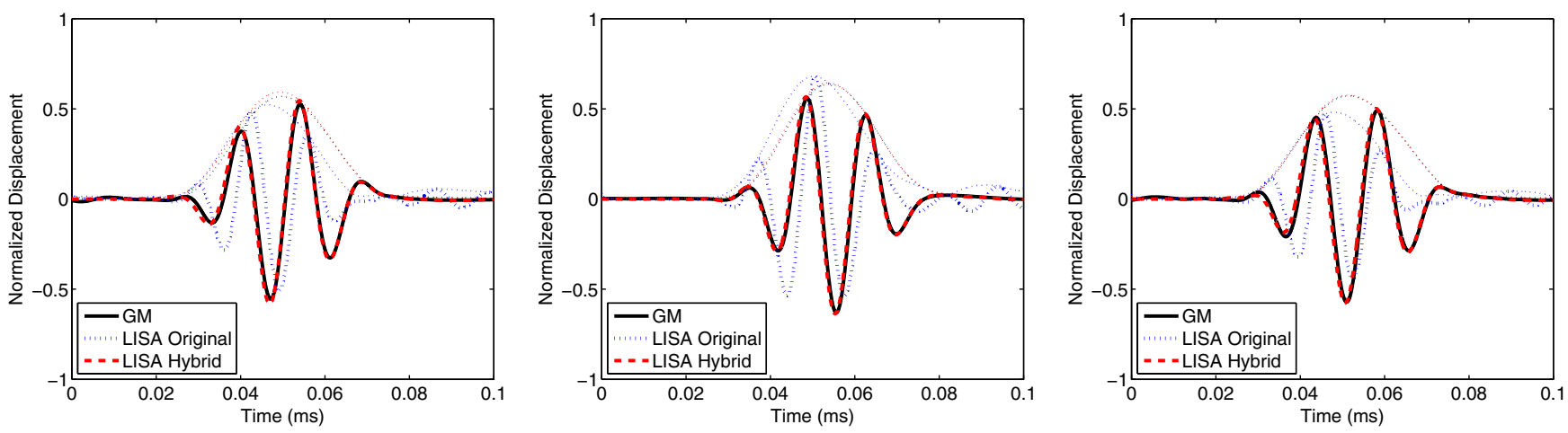

Fig. 25 Time history comparison for a cross-ply plate $40 \mathrm{~mm}$ from the square actuator center. The 0,45 , and 90 deg directions are shown from left to right.

propagating wave. For a given discretization and azimuthal direction, the group velocity error remained constant, as did the arrival time error of the propagating wave relative to the actual arrival time in the baseline model.

\section{B. Composite Long-Range Variable-Direction Emitting Radar Actua- tor Case}

The next actuator considered was the CLoVER transducer. For the models in this study, each sector of the actuator had an inside radius of $15 \mathrm{~mm}$ and an outside radius of $25 \mathrm{~mm}$. Each sector occupied a $45 \mathrm{deg}$ azimuthal region based around a center direction. For the aluminum plate, the 0 deg sector was active. Figure 19 shows the LISA and global matrix displacement time histories at points $70 \mathrm{~mm}$ from the center of the CLoVER profile at three different angles. As expected, the majority of the energy propagated in the direction corresponding to the active sector. For this case, the LISA original model produced displacement histories that were slightly out of phase with those from the global matrix model. Additionally, the LISA original model overpredicted the amplitudes present in the $90 \mathrm{deg}$ direction. These problems were not observed in the LISA hybrid model results.

The CLoVER actuator was also modeled with the cross-ply plate, and three different active sectors were simulated. Figures 20-22 show the results when the 0,45 , and 90 deg sectors were simulated. Only one sector was simulated at a time, and the other sectors were assumed to offer no contribution. Both the LISA original and LISA hybrid models correctly showed the directionality observed when the different sectors were used, but numerous discrepancies were seen in the LISA original results when they were closely compared to those from the global matrix model. Conversely, for each of the CLoVER sectors on the cross-ply plate, the LISA hybrid and global matrix results were almost indistinguishable.

For the cross-ply plate with a CLoVER actuator, directional dependence in the results was due to both the composite configuration and the shape of the actuator itself. The field plots shown in
Fig. 23 demonstrate the directionality in the out-of-plane displacement field produced when three different sectors are active. The figures on the left show the out-of-plane displacement observed $80 \mu$ s after the start of the actuation pulse using the global matrix method. The center figures show the LISA hybrid displacement field at the same instant in time. The figures on the right show the amplitude error of the LISA hybrid model compared to the global matrix results as a percentage of the maximum amplitude in the propagating wave. As in the case of the circular actuator, the amplitude errors in the CLoVER results were consistenly less than $10 \%$ of the maximum amplitude.

\section{Square Actuator Case}

Considering the previous results for the LISA original models and the actuation diagrams in Figs. 8 and 9 , it is evident the previous method of specifying in-plane displacements on the plate surface was not very successful in representing either the circular or CLoVER actuators. For these cases, the Cartesian nature of the LISA grid required that the model approximate the nonlinear profile of the actuators by locating the closest nodes. There was a clear mismatch between the nodes and the actuator boundary. In this section, results for a $10 \mathrm{~mm}$ square PZT actuator are presented. Unlike the previous cases, the square actuator can be exactly matched by the LISA discretization, avoiding any discrepancies associated with the discretization of the actuator in LISA. The next plots compare the two models and the global matrix results for the four plates under consideration.

The results for the aluminum laminate with the square actuator are shown in Fig. 24. As previously seen in the circular actuator case, the LISA original model produced results matching the baseline case fairly well, but not as closely as the LISA hybrid model. Again, the LISA original model seemed to produce improved results at distances far from the actuator, whereas the LISA hybrid model was reliable at each of the distances considered. 

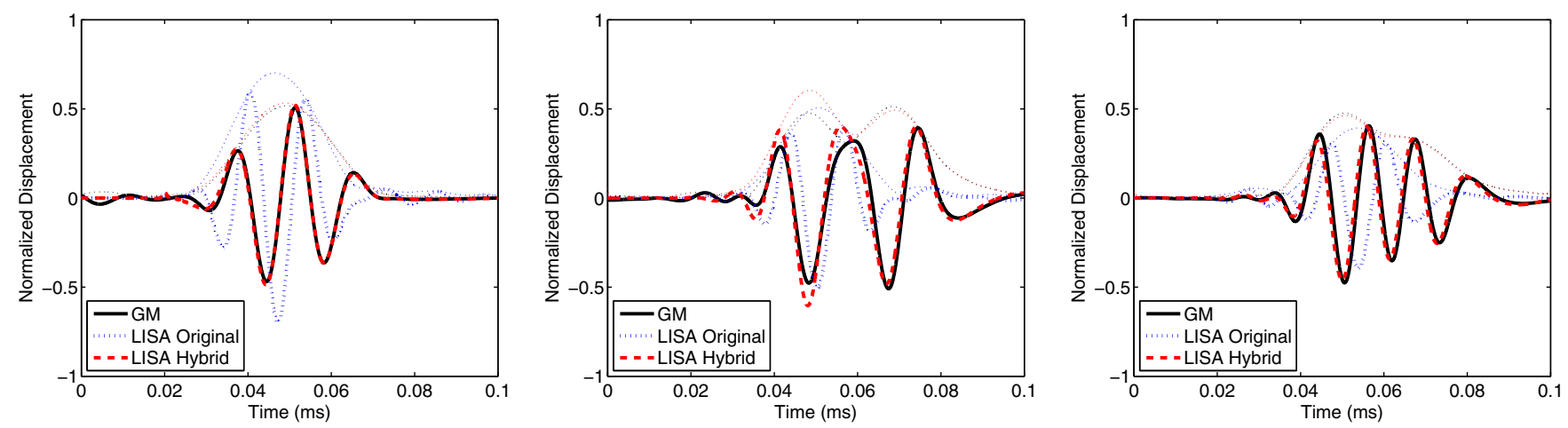

Fig. 26 Time history comparison for a unidirectional plate $40 \mathrm{~mm}$ from the square actuator center. The 0,45 , and 90 deg directions are shown from left to right.
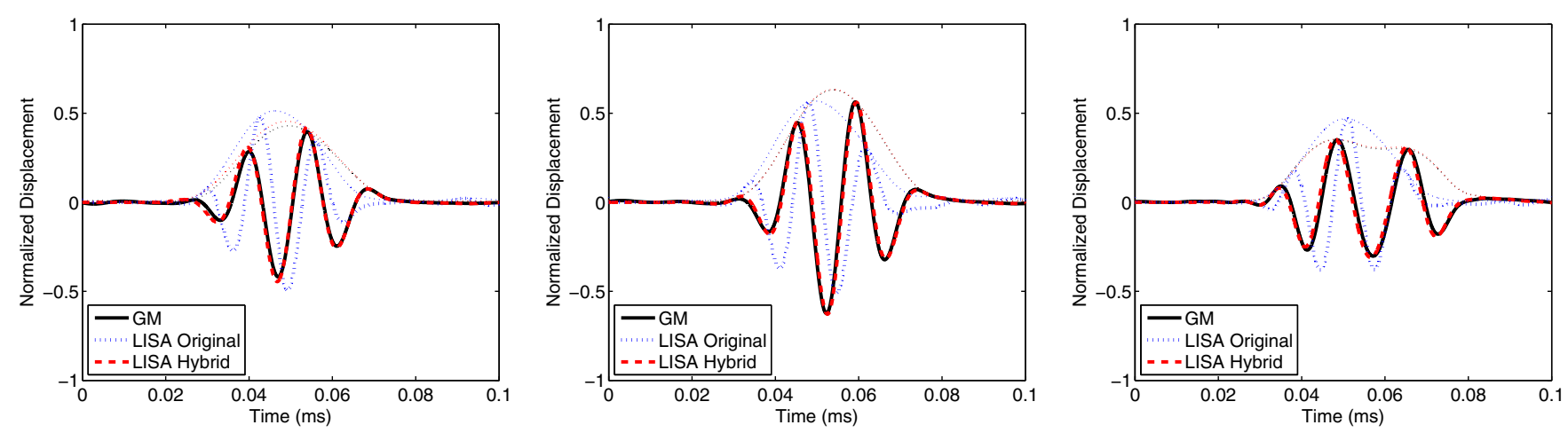

Fig. 27 Time history comparison for a quasi-isotropic plate $40 \mathrm{~mm}$ from the square actuator center. The 0,45 , and 90 deg directions are shown from left to right.

For the three composite models, shown in Figs. 25-27, the LISA hybrid model clearly outperformed the LISA original model at all of the angles that were considered. The results for each of the three layup sequences showed a clear mismatch between the global matrix model and the LISA original model.

For this square actuator case, modeling the actuator profile in the LISA orginal model was not a challenge because the Cartesian structure of the LISA discretization fits well with the actuator shape. However, the results showed that, even with this problem not being a factor, assuming a displacement field to represent the actuator did not produce satisfactory results. Only for the aluminum plate did the LISA orginal model produce results that came close to replicating the theoretical predictions. For the composite cases considered, the use of in-plane displacements to model the actuator was not a reliable method, even when the actuator fit well within the LISA grid scheme. Therefore, the actuator representation by prescribing displacements in the actuator region provides an additional source of error. This is the topic of study for a future paper.

\section{Conclusions}

This paper introduced the LISA hybrid methodology to solve for wave generation and propagation in isotropic and composite structures. It takes advantage of the strengths of the global matrix method and LISA. The global matrix method was used to determine the effects of piezoelectric actuators mounted on the surface of a plate. The LISA formulation for composite plates with plies in nonprincipal directions was used to capture the wave propagation away from the actuator. The LISA hybrid model was used to produce displacement results for an aluminum plate and three graphite-epoxy plates (cross-ply, unidirectional, and quasi-isotropic). These results were compared with baseline results from the global matrix method. In all cases, the LISA hybrid results produced more accurate time histories than previous LISA models, and the results matched those from the global matrix method extremely well. A brief study on the spatial discretization used in the LISA hybrid model showed the importance of this parameter and its effect on the resulting displacement time histories. Consistent with previous work, coarse discretizations produced artificially fast propagating waves. Additionally, this work showed the influence of the azimuthal direction on the discretization effects. For all four models, a $(1 / 6) t \times(1 / 6) t \times$ $(1 / 12) t$ cell size produced results agreeing well with the baseline model for plates of thickness $t$ when actuated with a center frequency producing a frequency-thickness product of $112.5 \mathrm{kHz}-\mathrm{mm}$.

Results from this work highlighted the importance of correctly modeling the actuator in numerical wave propagation simulations. The inability of the LISA orginal model to match the performance of the LISA hybrid model in the circular and CLoVER actuator cases illustrated the challenge of capturing nonrectangular actuators with the Cartesian grid in LISA. However, the results from the square actuator case also showed that, even when the actuator geometry can be exactly captured in the Cartesian grid, the assumption of prescribed in-plane displacements within the actuator profile does not adequately describe the behavior of the laminate. This work showed that the LISA hybrid model overcame both of these challenges, and it was effective for both simple PZT actuators and more complex piezocomposite actuators such as CLoVER. With the LISA hybrid model producing accurate results for the pristine plates considered in this work, future studies will involve using the model to consider various types of damage likely to be seen in aerospace structures.

\section{Appendix: Iterative Equations}

LISA iterative equations as derived by Nadella and Cesnik [14], which allow for in-plane rotation of the orthotropic lamina: 


$$
\begin{aligned}
& u^{i, j, k, t+1}=-u^{i, j, k, t-1}+2 u^{i, j, k}-\frac{2 \chi}{8} u^{i, j, k} \sum_{\alpha, \beta, \gamma= \pm 1}\left[\left(\eta_{x}^{2} \tilde{S}_{11}+\eta_{y}^{2} \tilde{S}_{66}+\eta_{z}^{2} \tilde{S}_{55}\right)\right] \\
& +\frac{\chi}{8} \sum_{\alpha, \beta, \gamma= \pm 1}\left[2 \eta_{x}^{2} \tilde{S}_{11} u^{i+\alpha, j, k}+2 \eta_{y}^{2} \tilde{S}_{66} u^{i, j+\beta, k}+2 \eta_{z}^{2} \tilde{S}_{55} u^{i, j, k+\gamma}\right] \\
& +\frac{\chi}{8} \sum_{\alpha, \beta, \gamma= \pm 1}\left[\alpha \beta \eta_{x} \eta_{y}\left(\tilde{S}_{12}+\tilde{S}_{66}\right)\left(v^{i+\alpha, j+\beta, k}-v^{i, j, k}\right)\right]+\frac{\chi}{8} \sum_{\alpha, \beta, \gamma= \pm 1}\left[\alpha \beta \eta_{x} \eta_{y}\left(\tilde{S}_{12}-\tilde{S}_{66}\right)\left(v^{i, j+\beta, k}-v^{i+\alpha, j, k}\right)\right] \\
& +\frac{\chi}{8} \sum_{\alpha, \beta, \gamma= \pm 1}\left[\alpha \gamma \eta_{x} \eta_{z}\left(\tilde{S}_{13}+\tilde{S}_{55}\right)\left(w^{i+\alpha, j, k+\gamma}-w^{i, j, k}\right)\right]+\frac{\chi}{8} \sum_{\alpha, \beta, \gamma= \pm 1}\left[\alpha \gamma \eta_{x} \eta_{z}\left(\tilde{S}_{13}-\tilde{S}_{55}\right)\left(w^{i, j, k+\gamma}-w^{i+\alpha, j, k}\right)\right] \\
& -\frac{2 \chi}{8} \sum_{\alpha, \beta, \gamma= \pm 1}\left[\alpha \beta \eta_{x} \eta_{y} \tilde{S}_{16}\left(u^{i, j, k}-u^{i+\alpha, j+\beta, k}\right)\right]-\frac{2 \chi}{8} v^{i, j, k} \sum_{\alpha, \beta, \gamma= \pm 1}\left[\eta_{x}^{2} \tilde{S}_{16}+\eta_{y}^{2} \tilde{S}_{26}\right] \\
& -\frac{\chi}{8} \sum_{\alpha, \beta, \gamma= \pm 1}\left[\beta \gamma \eta_{y} \eta_{z}\left(\tilde{S}_{36}+\tilde{S}_{45}\right) w^{i, j, k}\right]+\frac{2 \chi}{8} \sum_{\alpha, \beta, \gamma= \pm 1}\left[\eta_{x}^{2} \tilde{S}_{16} v^{i+\alpha, j, k}+\eta_{y}^{2} \tilde{S}_{26} v^{i, j+\beta, k}\right] \\
& +\frac{\chi}{8} \sum_{\alpha, \beta, \gamma= \pm 1}\left[\beta \gamma \eta_{y} \eta_{z} \tilde{S}_{36}\left(w^{i, j+\beta, k+\gamma}+w^{i, j, k+\gamma}-w^{i, j+\beta, k}\right)\right] \\
& +\frac{\chi}{8} \sum_{\alpha, \beta, \gamma= \pm 1}\left[\beta \gamma \eta_{y} \eta_{z} \tilde{S}_{45}\left(w^{i, j+\beta, k+\gamma}-w^{i, j, k+\gamma}+w^{i, j+\beta, k}\right)\right]+\frac{2 \chi}{8} \sum_{\alpha, \beta, \gamma= \pm 1} \eta_{z}^{2} \tilde{S}_{45}\left(v^{i, j, k+\gamma}-v^{i, j, k}\right) \\
& v^{i, j, k, t+1}=-v^{i, j, k, t-1}+2 v^{i, j, k} \\
& -\frac{2 \chi}{8} v^{i, j, k} \sum_{\alpha, \beta, \gamma= \pm 1}\left[\eta_{x}^{2} \tilde{S}_{66}+\eta_{y}^{2} \tilde{S}_{22}+\eta_{z}^{2} \tilde{S}_{44}\right]+\frac{\chi}{8} \sum_{\alpha, \beta, \gamma= \pm 1}\left[2 \eta_{x}^{2} \tilde{S}_{66} v^{i+\alpha, j, k}+2 \eta_{y}^{2} \tilde{S}_{22} v^{i, j+\beta, k}+2 \eta_{z}^{2} \tilde{S}_{44} v^{i, j, k+\gamma}\right] \\
& +\frac{\chi}{8} \sum_{\alpha, \beta, \gamma= \pm 1}\left[\alpha \beta \eta_{x} \eta_{y}\left(\tilde{S}_{12}+\tilde{S}_{66}\right)\left(u^{i+\alpha, j+\beta, k}-u^{i, j, k}\right)\right]+\frac{\chi}{8} \sum_{\alpha, \beta, \gamma= \pm 1}\left[\alpha \beta \eta_{x} \eta_{y}\left(\tilde{S}_{12}-\tilde{S}_{66}\right)\left(u^{i+\alpha, j, k}-u^{i, j+\beta, k}\right)\right] \\
& +\frac{\chi}{8} \sum_{\alpha, \beta, \gamma= \pm 1}\left[\beta \gamma \eta_{y} \eta_{z}\left(\tilde{S}_{23}+\tilde{S}_{44}\right)\left(w^{i, j+\beta, k+\gamma}-w^{i, j, k}\right)\right]+\frac{\chi}{8} \sum_{\alpha, \beta, \gamma= \pm 1}\left[\beta \gamma \eta_{y} \eta_{z}\left(\tilde{S}_{23}-\tilde{S}_{44}\right)\left(w^{i, j, k+\gamma}-w^{i, j+\beta, k}\right)\right] \\
& -\frac{2 \chi}{8} \sum_{\alpha, \beta, \gamma= \pm 1}\left[\alpha \beta \eta_{x} \eta_{y} \tilde{S}_{26}\left(v^{i, j, k}-v^{i+\alpha, j+\beta, k}\right)\right]-\frac{2 \chi}{8} u^{i, j, k} \sum_{\alpha, \beta, \gamma= \pm 1}\left[\eta_{x}^{2} \tilde{S}_{16}+\eta_{y}^{2} \tilde{S}_{26}\right] \\
& +\frac{2 \chi}{8} \sum_{\alpha, \beta, \gamma= \pm 1}\left[\eta_{x}^{2} \tilde{S}_{16} u^{i+\alpha, j, k}+\eta_{y}^{2} \tilde{S}_{26} u^{i, j+\beta, k}\right]-\frac{\chi}{8} \sum_{\alpha, \beta, \gamma= \pm 1}\left[\alpha \gamma \eta_{x} \eta_{z}\left(\tilde{S}_{36}+\tilde{S}_{45}\right) w^{i, j, k}\right] \\
& +\frac{\chi}{8} \sum_{\alpha, \beta, \gamma= \pm 1}\left[\alpha \gamma \eta_{x} \eta_{z} \tilde{S}_{36}\left(w^{i+\alpha, j, k+\gamma}+w^{i, j, k+\gamma}-w^{i+\alpha, j, k}\right)\right] \\
& +\frac{\chi}{8} \sum_{\alpha, \beta, \gamma= \pm 1}\left[\alpha \gamma \eta_{x} \eta_{z} \tilde{S}_{45}\left(w^{i+\alpha, j, k+\gamma}-w^{i, j, k+\gamma}+w^{i+\alpha, j, k}\right)\right]+\frac{2 \chi}{8} \sum_{\alpha, \beta, \gamma= \pm 1}\left[\eta_{z}^{2} \tilde{S}_{45}\left(u^{i, j, k+\gamma}-u^{i, j, k}\right)\right] \\
& w^{i, j, k, t+1}=-w^{i, j, k, t-1}+2 w^{i, j, k} \\
& -\frac{2 \chi}{8} w^{i, j, k} \sum_{\alpha, \beta, \gamma= \pm 1}\left[\eta_{x}^{2} \tilde{S}_{55}+\eta_{y}^{2} \tilde{S}_{44}+\eta_{z}^{2} \tilde{S}_{33}\right]+\frac{\chi}{8} \sum_{\alpha, \beta, \gamma= \pm 1}\left[2 \eta_{x}^{2} \tilde{S}_{55} w^{i+\alpha, j, k}+2 \eta_{y}^{2} \tilde{S}_{44} w^{i, j+\beta, k}+2 \eta_{z}^{2} \tilde{S}_{33} w^{i, j, k+\gamma}\right] \\
& +\frac{\chi}{8} \sum_{\alpha, \beta, \gamma= \pm 1}\left[\beta \gamma \eta_{y} \eta_{z}\left(\tilde{S}_{23}+\tilde{S}_{44}\right)\left(v^{i, j+\beta, k+\gamma}-v^{i, j, k}\right)\right]+\frac{\chi}{8} \sum_{\alpha, \beta, \gamma= \pm 1}\left[\beta \gamma \eta_{y} \eta_{z}\left(\tilde{S}_{23}-\tilde{S}_{44}\right)\left(v^{i, j+\beta, k}-v^{i, j, k+\gamma}\right)\right] \\
& +\frac{\chi}{8} \sum_{\alpha, \beta, \gamma= \pm 1}\left[\alpha \gamma \eta_{x} \eta_{z}\left(\tilde{S}_{13}+\tilde{S}_{55}\right)\left(u^{i+\alpha, j, k+\gamma}-u^{i, j, k}\right)\right]+\frac{\chi}{8} \sum_{\alpha, \beta, \gamma= \pm 1}\left[\alpha \gamma \eta_{x} \eta_{z}\left(\tilde{S}_{13}-\tilde{S}_{55}\right)\left(u^{i+\alpha, j, k}-u^{i, j, k+\gamma}\right)\right] \\
& -\frac{\chi}{8} \sum_{\alpha, \beta, \gamma= \pm 1}\left[\beta \gamma \eta_{y} \eta_{z}\left(\tilde{S}_{36}+\tilde{S}_{45}\right)\left(u^{i, j, k}-u^{i, j+\beta, k+\gamma}\right)\right]-\frac{\chi}{8} \sum_{\alpha, \beta, \gamma= \pm 1}\left[\alpha \gamma \eta_{x} \eta_{z}\left(\tilde{S}_{36}+\tilde{S}_{45}\right)\left(v^{i, j, k}-v^{i+\alpha, j, k+\gamma}\right)\right] \\
& -\frac{\chi}{8} \sum_{\alpha, \beta, \gamma= \pm 1}\left[\beta \gamma \eta_{y} \eta_{z}\left(\tilde{S}_{36}-\tilde{S}_{45}\right)\left(u^{i, j, k+\gamma}-u^{i, j+\beta, k}\right)\right]-\frac{\chi}{8} \sum_{\alpha, \beta, \gamma= \pm 1}\left[\alpha \gamma \eta_{x} \eta_{z}\left(\tilde{S}_{36}-\tilde{S}_{45}\right)\left(v^{i, j, k+\gamma}-v^{i+\alpha, j, k}\right)\right] \\
& +\frac{2 \chi}{8} \sum_{\alpha, \beta, \gamma= \pm 1}\left[\alpha \beta \eta_{x} \eta_{y} \tilde{S}_{45}\left(w^{i+\alpha, j+\beta, k}-w^{i, j, k}\right)\right] \\
& \eta_{x}=1 / \Delta x_{1} \\
& \eta_{y}=1 / \Delta x_{2} \\
& \eta_{z}=1 / \Delta x_{3} \\
& \chi=\Delta t^{2} / \rho
\end{aligned}
$$

In these equations, the current time step is assumed when it is not specifically mentioned. Stiffness terms with a tilde, such as $\tilde{S}_{11}$, indicate that the value of that term is defined by the value for one of the eight surrounding cells, depending on the current value of $\alpha, \beta, \gamma$.

\section{Acknowledgments}

This work was sponsored by the National Rotorcraft Technology Center Vertical Lift/Rotorcraft Center of Excellence at the University of Michigan, with Mahendra J. Bhagwat as the technical monitor. Opinions, interpretations, conclusions, and recommendations are 
those of the authors and are not necessarily endorsed by the U.S. Government.

\section{References}

[1] Raghavan, A., and Cesnik, C. E. S., "Review of Guided Wave Structural Health Monitoring," Shock and Vibration Digest, Vol. 39, No. 2, 2007, pp. 91-114. doi: $10.1177 / 0583102406075428$

[2] Ditri, J. J., and Rose, J. L., "Excitation of Guided Waves in Generally Anisotropic Layers Using Finite Sources," Journal of Applied Mechanics, Vol. 61, No. 2, 1994, pp. 330-338. doi: $10.1115 / 1.2901449$

[3] Datta, S. K., Shah, A. H., Bratton, R. L., and Chakraborty, T., "Wave Propagation in Laminated Composite Plates," Journal of the Acoustical Society of America, Vol. 83, No. 6, 1988, pp. 2020-2026. doi:10.1121/1.396382

[4] Lowe, M. J. S., "Matrix Techniques for Modeling Ultrasonic Waves in Multilayered Media," IEEE Transactions on Ultrasonics, Ferroelectrics, and Frequency Control, Vol. 42, No. 4, 1995, pp. 525-542. doi: $10.1109 / 58.393096$

[5] Lih, S. S., and Mal, A. K., "On the Accuracy of Approximate Plate Theories for Wave Field Calculations in Composite Laminates," Wave Motion, Vol. 21, No. 1, 1995, pp. 17-34. doi:10.1016/0165-2125(94)00038-7

[6] Raghavan, A., and Cesnik, C. E. S., "Modeling of Guided-Wave Excitation by Finite-Dimensional Piezoelectric Transducers in Composite Plates," 48th AIAA/ASME/ASCE/AHS/ASC Structures, Structural Dynamics, and Materials Conference, AIAA Paper 20071725, April 2007.

[7] Nadella, K. S., Salas, K. I., and Cesnik, C. E. S., "Characterization of Guided-Wave Propagation in Composite Plates," Proceedings of the SPIE, Health Monitoring of Structural and Biological Systems 2010, Vol. 7650, The International Society for Optical Engineering, San Diego, CA, 2010. doi: $10.1117 / 12.847887$

[8] Sundararaman, S., and Adams, D. E., "Modeling Guided Waves for Damage Identification in Isotropic and Orthotropic Plates Using a Local Interaction Simulation Approach," Journal of Vibration and Acoustics, Vol. 130, No. 4, 2008, Paper 041009. doi: $10.1115 / 1.2890389$

[9] Lee, B. C., and Staszewski, W. J., "Modeling of Lamb Waves for Damage Detection in Metallic Structures: Part 1. Wave Propagation," Smart Materials and Structures, Vol. 12, No. 5, 2003, pp. 804-814. doi:10.1088/0964-1726/12/5/018

[10] Delsanto, P. P., Whitcombe, T., Chaskelis, H. H., and Mignogna, R. B., "Connection Machine Simulation of Ultrasonic Wave Propagation in Materials 1: The One-Dimensional Case," Wave Motion, Vol. 16, No. 1, 1992 , pp. $65-80$. doi:10.1016/0165-2125(92)90047-6

[11] Delsanto, P. P., Schechter, R. S., Chaskelis, H. H., Mignogna, R. B., and Kline, R., "Connection Machine Simulation of Ultrasonic Wave
Propagation in Materials 2: The Two-Dimensional Case," Wave Motion, Vol. 20, No. 4, 1994, pp. 295-314. doi:10.1016/0165-2125(94)90016-7

[12] Delsanto, P. P., Schechter, R. S., and Mignogna, R. B., "Connection Machine Simulation of Ultrasonic Wave Propagation in Materials 3: The Three-Dimensional Case," Wave Motion, Vol. 26, No. 4, 1997, pp. 329-339. doi:10.1016/S0165-2125(97)00013-9

[13] Lee, B. C., and Staszewski, W. J., "Modeling of Lamb Waves for Damage Detection in Metallic Structures: Part II. Wave Interactions with Damage," Smart Materials and Structures, Vol. 12, No. 5, 2003, pp. 815-824. doi:10.1088/0964-1726/12/5/019

[14] Nadella, K. S., and Cesnik, C. E. S., "Numerical Simulation of Wave Propagation in Composite Plates," Proceedings of the SPIE, Health Monitoring of Structural and Biological Systems 2012, Vol. 8348, The International Society for Optical Engineering, San Diego, CA, 2012.

[15] Banerjee, S., Prosser, W., and Mal, A., "Calculation of the Response of a Composite Plate to Localized Dynamic Surface Loads Using a New wavenumber Integral Method," Journal of Applied Mechanics, Vol. 72, No. 1, 2005, pp. 18-24. doi:10.1115/1.1828064

[16] Salas, K. I., and Cesnik, C. E. S., "Guided Wave Excitation by a CLoVER Transducer for Structural Health Monitoring: Theory and Experiments," Smart Materials and Structures, Vol. 18, No. 7, 2009. doi:10.1088/0964-1726/18/7/075005.

[17] Sinor, M., "Numerical Modeling and Visualization of Elastic Waves Propagation in Arbitrary Complex Media," Proceedings of the 8th Workshop on Multimedia in Physics Teaching and Learning of the European Physical Society, Graz, 2004.

[18] Lee, B. C., and Staszewski, W. J., "Lamb Wave Propagation Modeling for Damage Detection: 1. Two-Dimensional Analysis," Smart Materials and Structures, Vol. 16, No. 2, 2007, pp. 249-259. doi:10.1088/0964-1726/16/2/003

[19] Lee, B. C., and Staszewski, W. J., "Lamb Wave Propagation Modeling for Damage Detection: 2. Damage Monitoring Strategy," Smart Materials and Structures, Vol. 16, No. 5, 2003, pp. 260-274.

[20] Sundararaman, S., and Adams, D. E., "Accuracy and Convergence Using a Local Interaction Simulation Approach in One, Two, and Three Dimensions," Journal of Applied Mechanics, Vol. 76, No. 3, 2009, Paper 031008. doi:10.1115/1.2871105

[21] Nadella, K. S., and Cesnik, C. E. S., "Simulation of Guided Wave Propagation in Isotropic and Composite Structures Using LISA," Proceedings of the 53rd AIAA/ASME/ASCE/AHS/ASC Structures, Structural Dynamics, and Materials Conference, AIAA Paper 20121387, April 2012.

R. Kapania Associate Editor 
This article has been cited by:

1. Rafal Radecki, Zhongqing Su, Li Cheng, Pawel Packo, Wieslaw J. Staszewski. 2018. Modelling nonlinearity of guided ultrasonic waves in fatigued materials using a nonlinear local interaction simulation approach and a spring model. Ultrasonics 84, 272-289. [Crossref]

2. Yanfeng Shen, Carlos E.S. Cesnik. 2017. Modeling of nonlinear interactions between guided waves and fatigue cracks using local interaction simulation approach. Ultrasonics 74, 106-123. [Crossref]

3. Yanfeng Shen, Carlos E S Cesnik. 2016. Hybrid local FEM/global LISA modeling of damped guided wave propagation in complex composite structures. Smart Materials and Structures 25:9, 095021. [Crossref] 